\title{
Intrinsic characteristic classes of a local Lie group
}

\author{
Ender Abadoğlu, Ercüment Ortaçgil
}

October 31, 2018

\begin{abstract}
For a local Lie group $M$ we define cohomology classes $\left[w_{2 k+1}\right] \in$ $H_{d R}^{2 k+1}(M, \mathbb{R})$. We show that $\left[w_{1}\right]$ is an obstruction to globalizability and give an example where $\left[w_{1}\right] \neq 0$. We also show that $\left[w_{3}\right]$ coincides with Godbillon-Vey class in a particular case. These classes are secondary as they emerge when curvature vanishes.
\end{abstract}

\section{Introduction}

The problem studied in this paper emerged from a general framework which we will outline in the next two paragraphs.

Let $M$ be a differentiable manifold with $\operatorname{dim} M=n \geq 2$. Let $p, q \in M$ and $j_{k}(f)^{p, q}$ be the $k$-jet of a local diffeomorphism $f$ with $f(p)=q$. We call $j_{k}(f)^{p, q}$ a $k$-arrow (from $p$ to $q$ ). A 0 -arrow is an ordered pair $(p, q)$. Now let $G$ be a connected Lie group which acts effectively on $G / H$. Following a program outlined in [21], we defined in [1] the geometric order $m$ of the (global) Klein geometry $G / H: G / H$ has order $m$, if any $g \in G$, as a transformation of $G / H$, is determined by any of its $m$-arrows and $m$ is the smallest such integer. Therefore $m=0$ eeeeeeeiff $H=\{e\}$. For any integer $m \geq 0$, there exists a compact Klein geometry $G / H$ of order $m$. In fact, we can choose $G \subset S L(m, \mathbb{R})$ and $H=$ the Borel subgroup of upper triangular matrices ([1]). If $\Delta \subset G$ is a discrete subgroup with $\Delta \cap H=\{e\}$, we obtain a pseudogroup on the discrete quotient $\Delta \backslash G / H$ with the property that its local diffeomorphisms are determined on their domains by any of their $m$-arrows. In this way we obtain pseudogroups which are finer than affine or projective pseudogroups. These pseudogroups exist even on Riemann surfaces but they are always subordinate to an affine or projective structure in this case.

Abstracting the arrows of the action of $G$ on $G / H$, we introduced in [22] the concept of a pre-homogeneous geometric structure of order $m$ on a manifold $M$ and the curvature of such a structure. For $m=0$, such a structure is absolute parallelism studied in this paper. Riemannian structures arise for $m=1$ by abstracting the 1 -arrows of the action of $S O(n) \propto \mathbb{R}^{n}$ on $\left(S O(n) \propto \mathbb{R}^{n}\right) / S O(n)=\mathbb{R}^{n}$. If $G / H$ has order $m$, then $g$ is determined also by 
its $(m+1)$-arrows. This fact implies the existence of some canonical splittings and associates some canonical "torsionfree connections" to such structures. As in this paper, curvature vanishes if and only if $(m+1)$-arrows integrate to a pseudogroup on $M$. If further the geometry is complete, then $M$ becomes a discrete quotient of a global model $G / H$ (see Proposition 15). Therefore, curvature is a measure of how much the geometry deviates from some local homogeneous model. This way of looking at curvature parallels the one proposed in the recent works [2], [3].

This paper is motivated by our attempt to do something globally interesting with the curvature in $[22]$ for $m=0$. This simplest case incorporates all the the main ideas and technical aspects of the above approach. The main technical result of this paper is the construction of certain secondary characteristic classes in the Lie algebra cohomology (therefore also in the de Rham cohomology) of a local Lie group $M$. It turns out that the first two classes are actually well known: $w_{1}$ is the character of the adjoint representation and therefore $\left[w_{1}\right]$ is an obstruction to unimodularity. We show that $\left[w_{1}\right]$ is also an obstruction to globalizability. The condition $w_{3}=0$ is the well known Cartan's criterion for solvability and the class $\left[w_{3}\right]$ is constructed first in [5] to show the nonvanishing of the third cohomology group of a semisimple Lie algebra (see Proposition 29 ). We find it surprising that $\left[w_{1}\right],\left[w_{3}\right]$ have not been viewed so far, to our knowledge, as the first two of a sequence of secondary characteristic classes in the literature. On the other hand, it is shown in [20] that the theory of local Lie groups is not a simple consequence of the global theory, but has its own set of interesting and delicate geometric structures. In this direction, we show here that it is possible to free the concept of a local Lie group from being local in such a way that it contains the theory of global Lie groups as a special case.

This paper is organized as follows. In Section 2 we recall the bracket [, ] on sections of $J_{1} T=$ the algebroid of $\mathcal{U}_{1}$ as defined in [17], [23], [24] and compute the exterior derivative of 1 -forms explicitly in local coordinates.

In Section 3 we formulate the existence of a parallelism on $M$ as a geometric structure defined by a splitting $\varepsilon$ of the groupoid projection $\pi: \mathcal{U}_{1} \rightarrow \mathcal{U}_{0}$.

The splitting $\varepsilon: \mathcal{U}_{0} \rightarrow \mathcal{U}_{1}$ determines two splittings $\widetilde{\Gamma}, \widehat{\Gamma}: T \rightarrow J_{1} T$ on the level of algebroids. The pair $(\widetilde{\Gamma}, \widehat{\Gamma})$ is a dual pair of affine connections as defined in [2], [3]. In Section 4 we associate two curvatures $\mathcal{R}_{1}(\varepsilon), \mathcal{R}_{2}(\varepsilon)$ to $\varepsilon$ and compute them explicitly in coordinates. We have $\mathcal{R}_{1}(\varepsilon)=0$ but not necessarily $\mathcal{R}_{2}(\varepsilon)=0$.

In Section 5 we construct a closed 1-form $\omega_{1}$ in the algebroid cohomology of $J_{1} T$ with trivial coefficients (see [18], [19], [6], [7], [11] for cohomology of Lie algebroids). Our local computations uncover the fundamental roles of the Spencer operator and the algebraic bracket in the construction of this secondary characteristic class. It seems to us that the construction of $\omega_{1}$ may be recast in the more general framework of secondary characteristic classes for Lie algebroids introduced recently in the works [6], [7], [11]. If this is the case, we hope that our approach will have something to add to these works. However, our main concern here is not $\omega_{1}$ but its restriction to $\widetilde{\Gamma}(T) \subset J_{1} T$ which pulls back to a 1-form $w$ in de Rham complex, not necessarily closed. We prove $d w_{1}=\operatorname{Tr} \mathcal{R}_{2}(\varepsilon)$ 
(Proposition 7). Our approach gives a totally new way of looking at torsion, a point emphasized first in [28] in a somewhat different setting.

In Section 6 we show that $\mathcal{R}_{2}(\varepsilon)=0$ if and only if $M$ is a local Lie group (Proposition 8). Therefore the 1 -form $w_{1}$ is closed on a local Lie group and the problem is to understand the obstruction to its exactness.

In Section 7 we define another curvature $\mathcal{R}(\varepsilon)$ which is the curvature mentioned in the second paragraph above. We show $\mathcal{R}(\varepsilon)=0 \Longleftrightarrow \mathcal{R}_{2}(\varepsilon)=0$. It turns out that the implication $\mathcal{R}_{2}(\varepsilon)=0 \Longrightarrow \mathcal{R}(\varepsilon)=0$ is equivalent to the traditional form of the third fundamental theorem of Lie. We construct an explicit local primitive of the closed form $w_{1}$ (Proposition 21). This fact implies the main result of this section (Proposition 22) that if $\left[w_{1}\right] \neq 0$ in $H_{d R}^{1}(M, \mathbb{R})$ then $M$ is not globalizable. We give an example where $\left[w_{1}\right] \neq 0$. Definitely, there is a relation between the cohomology class $\left[w_{1}\right]$ and the modular class introduced in [10] and studied further in [29]. As shown in [20], the concepts of globalizability and global associativity are equivalent for local Lie groups. Many explicit and nontrivial examples of local Lie groups are constructed in [20] which fail to be globalizable. One of these examples is even simply connected but this pathology can not occur if $M$ is complete in view of Corollary 19. We plan to take up the study of these examples in some future work.

In Section 8 we define the higher order analogs of the closed forms $\omega_{1}$ and $w_{1}$. If $G=S L(2, \mathbb{R}), \Delta \subset G$ a cocompact discrete subgroup and $\mathcal{F}$ is the standard codimension one foliation on the local Lie group $G / \Delta$, we show (Proposition $28)$ that $\left[w_{3}\right] \in H^{3}(G / \Delta, \mathbb{R})$ coincides with the Godbillon-Vey class $G V(\mathcal{F})$ and therefore $\left[w_{3}\right] \neq 0$ by the well known Roussarie calculation (see [4]). We find this example particulary interesting since our construction of $\left[w_{3}\right]$ is independent of any foliation. It is natural to expect that these higher classes are also obstructions to globalizability. We give an example, communicated to us by the referee, which shows that this expectation is unjustified.

Therefore, generalizing the construction of the characteristic classes in this paper to arbitrary geometric order $m$, showing their nontriviality and clarifying their geometric meaning remain as challenging problems.

\section{The algebroid $J_{1} T$}

Let $M$ be a (connected) differentiable manifold with $\operatorname{dim} M=n \geq 2$. Let $p, q \in M$ and $j_{k}(f)^{p, q}$ be the $k$-jet of a local diffeomorphism $f$ with $f(p)=q$. We call $j_{k}(f)^{p, q}$ a $k$-arrow (from $p$ to $q$ ). Note that a 0 -arrow is an ordered pair $(p, q)$. Let $\mathcal{U}_{k}^{p, q}$ denote the set of all $k$-arrows on $M$ from $p$ to $q$. We define the set $\mathcal{U}_{k} \stackrel{\text { def }}{=} \cup_{p, q \in M} \mathcal{U}_{k}^{p, q}$. We have the composition map $\mathcal{U}_{k}^{q, r} \times \mathcal{U}_{k}^{p, q} \rightarrow \mathcal{U}_{k}^{p, r}$ defined by $j_{k}(g)^{q, r} \circ j_{k}(f)^{p, q} \stackrel{\text { def }}{=} j_{k}(g \circ f)^{p, r}$. The differentiable structure on $M$ induces a differentiable structure on $\mathcal{U}_{k}$ and $\mathcal{U}_{k}$ is a transitive Lie equation (in finite form), which is a very special groupoid (see [17], [23], [24] for Lie equations in finite and infinitesimal forms and [18], [19] and the references therein for Lie groupoids and algebroids). Note that $\mathcal{U}_{0}$ is the pair groupoid $M \times M$. We have 
the projection map $\pi_{k, j}: \mathcal{U}_{k} \rightarrow \mathcal{U}_{j}, j \leq k+1$, induced by the projection of jets and $\pi_{k, j}$ is a morphism of groupoids, that is, it preserves composition and inversion of arrows. In this paper we need only $\mathcal{U}_{0}$ and $\mathcal{U}_{1}$.

Recall that the algebroid of $\mathcal{U}_{0}$ is the tangent bundle $T \rightarrow M$. We now recall the algebroid of $\mathcal{U}_{1}$ (see [23], [24] for further details). Let $J_{1} T \rightarrow M$ be the vector bundle whose fiber over $p \in M$ consists of 1 -jets of vector fields at $p$. We denote sections of $J_{1} T \rightarrow M$ by $X, Y$ and sections of $T \rightarrow M$ by $\xi, \eta$. We sometimes use the same notation for a section and its value at a point. We also use the same notation $E$ for both the total space of a vector bundle $E \rightarrow M$ and for the space of global sections of $E \rightarrow M$.

Now a section $X$ of $J_{1} T \rightarrow M$ over $\left(U, x^{i}\right)$ is of the form $\left(X^{i}(x), X_{j}^{i}(x)\right)$ and the projection $\pi: J_{1} T \rightarrow T$ is given by $\left(X^{i}, X_{j}^{i}\right) \rightarrow\left(X^{i}\right)$. We have the Spencer operator $D: J_{2} T \rightarrow T^{*} \otimes J_{1} T$ locally given by $\left(X^{i}, X_{j}^{i}, X_{j k}^{i}\right) \rightarrow\left(\partial_{j} X^{i}-\right.$ $\left.X_{j}^{i}, \partial_{k} X_{j}^{i}-X_{k j}^{i}\right)$. We have the algebraic bracket $\{,\}_{p}:\left(J_{2} T\right)_{p} \times\left(J_{2} T\right)_{p} \rightarrow$ $\left(J_{1} T\right)_{p}$ whose coordinate formula is obtained by differentiating the usual formula for the bracket of two vector fields twice, evaluating at $p$ and replacing derivatives with jet coordinates. Finally, we have the Spencer bracket [, ] on sections of $J_{1} T \rightarrow T$ defined by

$$
[X, Y]=\{\tilde{X}, \tilde{Y}\}+i_{\pi X} D(\widetilde{Y})-i_{\pi Y} D(\widetilde{X})
$$

In (1), $\tilde{X}, \tilde{Y}$ are arbitrary lifts of $X$ and $Y$ to sections of $J_{2} T$ and $i_{\pi X}$ denotes contraction with respect to the vector field $\pi X$. The bracket $[X, Y]$ does not depend on the lifts $\widetilde{X}, \widetilde{Y}$. If $X=\left(X^{i}, X_{j}^{i}\right)$ and $Y=\left(Y^{i}, Y_{j}^{i}\right)$, using (1) we compute

$$
\begin{aligned}
{[X, Y]^{i} } & =X^{a} \frac{\partial Y^{i}}{\partial x^{a}}-Y^{a} \frac{\partial X^{i}}{\partial x^{a}} \\
{[X, Y]_{j}^{i} } & =X_{j}^{a} Y_{a}^{i}-Y_{j}^{a} X_{a}^{i}+X^{a} \frac{\partial Y_{j}^{i}}{\partial x^{a}}-Y^{a} \frac{\partial X_{j}^{i}}{\partial x^{a}}
\end{aligned}
$$

where we use summation convention in (2). With the bracket given by (2), $J_{1} T \rightarrow T$ becomes the Lie algebroid of the Lie groupoid $\mathcal{U}_{1}$. Note that $\pi$ : $J_{1} T \rightarrow T$ becomes a homomorphism of algebroids in view of (2).

As shown in [7], [3], for any Lie algebroid $\mathcal{A}$, there is a unique Lie algebroid structure on $J_{1} \mathcal{A}$ compatible with $\mathcal{A}$. If $\mathcal{A}=T$, then the bracket of $J_{1} \mathcal{A}$ as defined in these works coincides with (2), even though the role of the Spencer operator is not evident at first sight.

We use the same notation [, ] for the brackets of $J_{1} T$ and $T$. For a vector field $\xi$, let $j_{1} \xi$ denote the first prolongation of $\xi$. In coordinates, if $\xi=\left(X^{i}\right)$, then $j_{1} \xi=\left(X^{i}, \frac{\partial X i}{\partial x^{j}}\right) \in J_{1} T$. We have

$$
j_{1}[\xi, \eta]=\left[j_{1} \xi, j_{1} \eta\right]
$$

that is, [ , ] respects prolongation. (3) is easily checked using (2). 
We now define a representation of $J_{1} T$ on $C^{\infty}(M)$ by $X(f) \stackrel{\text { def }}{=} \mathcal{L}_{\pi X}(f)$ where $\mathcal{L}_{\pi X}$ denotes Lie derivative with respect to the vector field $\pi X$, and consider the cohomology of $J_{1} T$ with respect to this representation (see [18], [19], [6], [7], [11] for cohomology of Lie algebroids). Up to Section 8, we will be interested only in 1-forms. Let $\left(J_{1} T\right)^{*} \rightarrow M$ be the dual bundle of $J_{1} T \rightarrow M$.

Definition $1 \mathrm{~A}$ 1-form (of the algebroid $J_{1} T$ ) is a (smooth) section of $\left(J_{1} T\right)^{*}$ $\rightarrow M$.

So a 1-form $\omega$ pairs linearly with sections of $J_{1} T$ to functions on $M$. The 1 -form $\omega$ is locally of the form $\left(\omega_{i}(x), \omega_{j}^{i}(x)\right)$ and the pairing is given by

$$
\omega(X) \stackrel{\text { def }}{=} X^{a}(x) \omega_{a}(x)+X_{b}^{a}(x) \omega_{a}^{b}(x)
$$

It is easy to derive the transformation laws of the components of $X$ and $\omega$ which we will not do here. These transformation laws show that $\left(X^{i}, 0\right)$ has no invariant meaning whereas $\left(0, X_{j}^{i}\right)$ does and belongs to $\operatorname{Ker}(\pi)$. In contrast, $\left(\omega^{i}, 0\right)$ does have invariant meaning and represents an ordinary 1-form as a cochain in the deRham complex whereas $\left(0, \omega_{j}^{i}\right)$ has no invariant meaning. Therefore $\omega$ reduces to an ordinary 1-form eeeee if and only if $\omega_{j}^{i}=0$.

Now we want to compute the exterior derivative $\delta(\omega)$ of $\omega$ in coordinates. Differentiating (4) and contracting with the vector field $\pi X$, we get

$$
\begin{aligned}
\mathcal{L}_{\pi X} \omega(Y)= & X^{c} \frac{\partial \omega(Y)}{\partial x^{c}}=X^{c} \frac{\partial Y^{a}}{\partial x^{c}} \omega_{a}+X^{c} Y^{a} \frac{\partial \omega_{a}}{\partial x^{c}} \\
& +X^{c} \frac{\partial Y_{b}^{a}}{\partial x^{c}} \omega_{a}^{b}+X^{c} Y_{b}^{a} \frac{\partial \omega_{a}^{b}}{\partial x^{c}}
\end{aligned}
$$

Interchanging $X$ and $Y$ in (5) and subtracting the resulting formula from (5), we get $\mathcal{L}_{\pi X} \omega(Y)-\mathcal{L}_{\pi Y} \omega(X)=$

$$
\begin{aligned}
& \left(X^{c} Y^{a}-Y^{c} X^{a}\right) \frac{\partial \omega_{a}}{\partial x^{c}}+\left(X^{c} Y_{b}^{a}-Y^{c} X_{b}^{a}\right) \frac{\partial \omega_{a}^{b}}{\partial x^{c}} \\
& -\left(X_{b}^{c} Y_{c}^{a}-Y_{b}^{c} X_{c}^{a}\right) \omega_{a}^{b}+\left(X^{c} \frac{\partial Y^{a}}{\partial x^{c}}-Y^{c} \frac{\partial X^{a}}{\partial x^{c}}\right) \omega_{a} \\
& +\left(X_{b}^{c} Y_{c}^{a}-Y_{b}^{c} X_{c}^{a}+X^{c} \frac{\partial Y_{b}^{a}}{\partial x^{c}}-Y^{c} \frac{\partial X_{b}^{a}}{\partial x^{c}}\right) \omega_{a}^{b}
\end{aligned}
$$

where we added and subtracted $\left(X_{b}^{c} Y_{c}^{a}-Y_{b}^{c} X_{c}^{a}\right) \omega_{a}^{b}$ in the LHS of (6). In view of (2) and (4), the sum of the last two terms in (6) is $\eta[X, Y]$ so that (6) becomes the well known formula

$$
\mathcal{L}_{\pi X} \omega(Y)-\mathcal{L}_{\pi Y} \omega(X)=\delta \omega(X, Y)+\omega[X, Y]
$$

where 


$$
\begin{aligned}
\delta \omega(X, Y)= & \left(X^{c} Y^{a}-Y^{c} X^{a}\right) \frac{\partial \omega_{a}}{\partial x^{c}}+\left(X^{c} Y_{b}^{a}-Y^{c} X_{b}^{a}\right) \frac{\partial \omega_{a}^{b}}{\partial x^{c}} \\
& -\left(X_{b}^{c} Y_{c}^{a}-Y_{b}^{c} X_{c}^{a}\right) \omega_{a}^{b}
\end{aligned}
$$

If $\omega_{j}^{i}=\delta_{j}^{i}$, note that the last term in (8) vanishes since $\operatorname{Tr}(a b)=\operatorname{Tr}(b a)$ where $\operatorname{Tr}$ denotes trace.

\section{$3 \quad$ Parallelism as splitting}

Definition 2 A splitting of $\pi_{1,0}: \mathcal{U}_{1} \rightarrow \mathcal{U}_{0}$ is a morphism of groupoids $\varepsilon: \mathcal{U}_{0} \rightarrow$ $\mathcal{U}_{1}$ such that $\pi_{1,0} \circ \varepsilon=i d$.

So a splitting $\varepsilon$ assigns to any ordered pair $(p, q)$ a 1-arrow from $p$ to $q$ and this assignment preserves composition and inversion of arrows. We will denote $\varepsilon(p, q)$ by $\varepsilon^{p, q}$. Further, $\varepsilon$ is differentiable and $\varepsilon\left(\mathcal{U}_{0}\right)$ is an imbedded submanifold of $\mathcal{U}_{1}$. Note that $\varepsilon^{p, p}=1$-arrow of the identity map at $p$. Also, a 1-arrow $f^{p, q}$ from $p$ to $q$ determines an isomorphism $\left(f^{p, q}\right)_{*}: T_{p} \rightarrow T_{q}$ and if two 1-arrows determine the same isomorphism, then they are identical. This observation supplies the proof of the following simple

Lemma 3 The following are equivalent:

i) There exists a splitting $\varepsilon: \mathcal{U}_{0} \rightarrow \mathcal{U}_{1}$

ii) $M$ is parallelizable

Henceforth, we always assume that $M$ is parallelizable with a splitting $\varepsilon$ which we fix once and for all. The splitting $\varepsilon$ is the analog of a torsionfree connection. Note that geometry $(=$ parallelism) and connection $(=\varepsilon)$ are identical objects in the present framework.

Let $\left(U, x^{i}\right),\left(V, y^{i}\right)$ be any two coordinate patches on $M$. The splitting $\varepsilon$ is of the form $\varepsilon_{j}^{i}(x, y)$ on $U \times V$. We call the differentiable functions $\varepsilon_{j}^{i}(x, y)$ the components of $\varepsilon$ on $U \times V$. The isomorphism $\left(\varepsilon^{x, y}\right)_{*}$ is given by $X^{i}(x) \rightarrow$ $\varepsilon_{j}^{i}(x, y) X^{j}(x)=Y^{i}(y)$ in coordinates. We will repeatedly use the local formulas $\varepsilon_{a}^{i}(y, z) \varepsilon_{j}^{a}(x, y)=\varepsilon_{j}^{i}(x, z), \varepsilon_{j}^{i}(x, x)=\delta_{j}^{i}$ in the following sections.

\section{The curvatures $\mathcal{R}_{1}(\varepsilon), \mathcal{R}_{2}(\varepsilon)$}

Let $p \in\left(U, x^{i}\right)$. We choose $X(p) \in T_{p}$ arbitrarily and define a vector field $\xi=\left(X^{i}\right)$ on $\left(U, x^{i}\right)$ by

$$
X^{i}(x) \stackrel{\text { def }}{=} \varepsilon_{a}^{i}(p, x) X^{a}(p)
$$

Definition $4 A$ vector field $\vartheta$ is called $\varepsilon$-invariant if $\left(\varepsilon^{p, q}\right)_{*} \vartheta(p)=\vartheta(q), p, q \in$ $M$. 
We denote the Lie algebra of vector fields on $M$ by $\mathfrak{X}(M)$ and the vector space of $\varepsilon$-invariant vector fields by $\mathfrak{X}_{\varepsilon}(M)$. Clearly $\xi \in \mathfrak{X}_{\varepsilon}(M)$ is uniquely determined by $\xi(p)$ for any $p \in M$ and therefore $\operatorname{dim} \mathfrak{X}_{\varepsilon}(M)=\operatorname{dim} M$. However, $\mathfrak{X}_{\varepsilon}(M)$ need not be a Lie algebra, that is, we may not have $\left[\mathfrak{X}_{\varepsilon}(M), \mathfrak{X}_{\varepsilon}(M)\right] \subset \mathfrak{X}_{\varepsilon}(M)$. Some $\xi=\left(X^{i}\right) \in \mathfrak{X}_{\varepsilon}(M)$ is given by $(9)$ on $\left(U, x^{i}\right)$.

Differentiating (9) and evaluating at $x=p$, we obtain

$$
\frac{\partial X^{i}}{\partial x^{j}}(p)=\left[\frac{\partial \varepsilon_{a}^{i}(p, x)}{\partial x^{j}}\right]_{x=p} X^{a}(p)
$$

We define

$$
\Gamma_{j k}^{i}(x) \stackrel{\text { def }}{=}\left[\frac{\partial \varepsilon_{k}^{i}(x, y)}{\partial y^{j}}\right]_{y=x}
$$

Note that $\Gamma_{j k}^{i}(x)$ need not be symmetric in $j, k$. This fact will be of fundamental importance below. (10), (11) show that $\Gamma$ defines a map of vector bundles

$$
\begin{aligned}
\widetilde{\Gamma}: & T \rightarrow J_{1} T \\
: & X^{i} \longrightarrow\left(X^{i}, \Gamma_{j a}^{i} X^{a}\right)
\end{aligned}
$$

We have the exact sequence

$$
0 \longrightarrow \operatorname{Ker}(\pi) \longrightarrow J_{1} T \stackrel{\pi}{\longrightarrow} T \longrightarrow 0
$$

where $\operatorname{Ker}(\pi) \simeq T^{*} \otimes T$. So $\pi \circ \widetilde{\Gamma}=i d_{T}$ and $\widetilde{\Gamma}$ defines a splitting of the extension (13).

Now let $\vartheta=\left(X^{i}\right)$ be a vector field. We have the $P D E$ defined on $\mathfrak{X}(M)$ by

$$
\begin{aligned}
j_{1}(\vartheta) & =\widetilde{\Gamma}(\vartheta) \\
\frac{\partial X^{i}}{\partial x^{j}} & =\Gamma_{j a}^{i} X^{a}
\end{aligned}
$$

We define $\mathcal{R}_{1}(\varepsilon)$ by

$$
\mathcal{R}_{1}(\varepsilon)_{r j, k}^{i} \stackrel{\text { def }}{=}\left[\frac{\partial \Gamma_{j k}^{i}}{\partial x^{r}}+\Gamma_{r k}^{a} \Gamma_{j a}^{i}\right]_{[r, j]}
$$

where $[r j]$ denotes alternation. It is easy to show that $\mathcal{R}_{1}(\varepsilon) \in \wedge^{2}\left(T^{*}\right) \otimes T^{*} \otimes T$. A straightforward computation using (14) shows that $\mathcal{R}_{1}(\varepsilon)=0$ is the integrability condition of (14). Equivalently, we can define $\nabla_{j} X^{i} \stackrel{\text { def }}{=} \frac{\partial X^{i}}{\partial x^{j}}-\Gamma_{j a}^{i} X^{a} \in T^{*} \otimes T$ which gives $\nabla_{r} \nabla_{j} X^{i}-\nabla_{j} \nabla_{r} X^{i}=\mathcal{R}_{1}(\varepsilon)_{r j, a}^{i} X^{a}$. Note that the sign of $\Gamma_{j a}^{i} X^{a}$ we use in $\nabla_{j} X^{i}$ is the opposite of the one in tensor calculus: we could define $\Gamma_{j k}^{i}(x)$ by $\left[\frac{\partial \varepsilon_{k}^{i}(x, y)}{\partial x^{j}}\right]_{y=x}$. Differentiating $\varepsilon_{a}^{i}(y, x) \varepsilon_{j}^{a}(x, y)=\delta_{j}^{i}$ and evaluating on diagonal we get $\left[\frac{\partial \varepsilon_{k}^{i}(x, y)}{\partial y^{j}}\right]_{y=x}=-\left[\frac{\partial \varepsilon_{k}^{i}(x, y)}{\partial x^{j}}\right]_{y=x}$. 
Lemma $5 \xi \in \mathfrak{X}(M)$ belongs to $\mathfrak{X}_{\varepsilon}(M)$ if and only if it is a solution of (14). In particular $\mathcal{R}_{1}(\varepsilon)=0$.

Proof: Since $p$ is arbitrary in (10), any $\xi \in \mathfrak{X}_{\varepsilon}(M)$ is a solution of (14). Therefore $\mathcal{R}_{1}(\varepsilon)=0$ by the definition of $\mathcal{R}_{1}(\varepsilon)$. Conversely, let $\vartheta \in \mathfrak{X}(M)$ be a solution of (14). We choose $p \in\left(U, x^{i}\right)$ and extend $\vartheta(p)$ to an $\varepsilon$-invariant $\xi$ on $M$. Now $\vartheta, \xi$ both solve (14) and have the same initial condition at $p$. By uniqueness, $\vartheta=\xi$ on some neighborhood $p \in \bar{U} \subset U$. Therefore, the set $A \stackrel{\text { def }}{=}\{p \in M \mid \vartheta(p)=\xi(p)\} \neq \emptyset$ is both open and closed in $M$ and we conclude $A=M$.

To clarify the relation between (12), (14), (15) and the formalism of connections on principal bundles, we now choose $p \in\left(U, x^{i}\right)$ and define

$$
\bar{\Gamma}_{j k}^{i}(p, x) \stackrel{\text { def }}{=} \frac{\partial \varepsilon_{b}^{i}(p, x)}{\partial x^{j}} \varepsilon_{k}^{b}(x, p)
$$

We claim that $\bar{\Gamma}_{j k}^{i}(p, x)$ is independent of $p$. Differentiating $\varepsilon_{a}^{i}(q, x) \varepsilon_{j}^{a}(p, q)=$ $\varepsilon_{j}^{i}(p, x)$, we obtain

$$
\frac{\partial \varepsilon_{a}^{i}(q, x)}{\partial x^{k}} \varepsilon_{j}^{a}(p, q)=\frac{\partial \varepsilon_{j}^{i}(p, x)}{\partial x^{k}}
$$

Multiplying (17) with $\varepsilon_{b}^{i}(q, p) \varepsilon_{j}^{b}(x, q)=\varepsilon_{j}^{i}(x, p)$ and summing over $j$ gives $\bar{\Gamma}_{j k}^{i}(q, x)=\bar{\Gamma}_{j k}^{i}(p, x)$ as claimed. Now setting $p=x$ in (16), we conclude

$$
\bar{\Gamma}_{j k}^{i}(p, x)=\Gamma_{j k}^{i}(x)
$$

We now fix $p \in M$ and a coordinate system around $p$ once and for all and consider the principal bundle $\mathcal{U}_{1}^{(p)} \stackrel{\text { def }}{=} \cup_{x \in M} \mathcal{U}^{p, x} \rightarrow M$ with structure group $\mathcal{U}^{p, p} \simeq G L(n, \mathbb{R})$. This principal bundle can be identified (not canonically) with the principal frame bundle of $M$. Now $\varepsilon$ trivializes this bundle as $x \rightarrow \varepsilon^{p, x}$ and therefore defines a flat connection with trivial monodromy. The components of this connection are given by (18) and its curvature is $\mathcal{R}_{1}(\varepsilon)$. However, the horizontal lift to the principal bundle $\mathcal{U}_{1}^{(p)} \rightarrow M$ is not given by (12) but by (21) below. We define the Lie algebra bundle $\mathcal{L} \stackrel{\text { def }}{=} \cup_{q \in M} \mathcal{L}\left(\mathcal{U}^{q, q}\right)$ where $\mathcal{L}\left(\mathcal{U}^{q, q}\right)$ denotes the Lie algebra of $\mathcal{U}^{q, q}$. Since $\mathcal{L} \simeq T^{*} \otimes T$, we have $\mathcal{R}_{1}(\varepsilon) \in \wedge^{2}\left(T^{*}\right) \otimes \mathcal{L}$. This flat connection determines a flat connection on the associated tangent bundle which is given by (12). This gives another reason (more familiar than Lemma 5) why we should have $\mathcal{R}_{1}(\varepsilon)=0$.

Now

$$
\Gamma_{j k}^{i}-\Gamma_{k j}^{i} \stackrel{\text { def }}{=} T_{j k}^{i}=\text { torsion }
$$

As for $\mathcal{R}_{2}(\varepsilon)$, following [21] (see also [3] for a different but equivalent definition) we define

$$
\mathcal{L}_{X} \xi \stackrel{\text { def }}{=}[\pi X, \xi]+i_{\xi} D(X)
$$


where $D: J_{1} T \rightarrow T^{*} \otimes T$ is the Spencer operator locally given by $\left(X^{i}, X_{j}^{i}\right) \rightarrow$ $\left(\frac{\partial X^{i}}{\partial x^{j}}-X_{j}^{i}\right)$. Therefore $\mathcal{L}_{X}: T \rightarrow T$ is a first order differential operator. To emphasize the analogy with $\mathcal{R}_{1}(\varepsilon)$, we denote $\mathcal{L}_{\Gamma\left(\frac{\partial}{\partial x^{i}}\right)}$ by $\widehat{\nabla}_{i}$. Using (2) and (20) we find $\widehat{\nabla}_{j} X^{i}=\frac{\partial X^{i}}{\partial x^{j}}-\Gamma_{a j}^{i} X^{a}$ which gives another splitting $\widehat{\Gamma}$ of (13) defined by

$$
\begin{aligned}
\widehat{\Gamma} \quad: & T \rightarrow J_{1} T \\
: & X^{i} \longrightarrow\left(X^{i}, \Gamma_{a j}^{i} X^{a}\right)
\end{aligned}
$$

If $T_{j k}^{i}=0$, then clearly $\widetilde{\Gamma}=\widehat{\Gamma}$. We define

$$
\mathcal{R}_{2}(\varepsilon)_{r j, k}^{i} \stackrel{\text { def }}{=}\left[\frac{\partial \Gamma_{k j}^{i}}{\partial x^{r}}+\Gamma_{k r}^{a} \Gamma_{a j}^{i}\right]_{[r, j]}
$$

and check that $\mathcal{R}_{2}(\varepsilon)=0$ is the integrability condition of $\frac{\partial X^{i}}{\partial x^{j}}=\Gamma_{a j}^{i} X^{a}$. It is also straightforward to check $\widetilde{\nabla}_{r} \widetilde{\nabla}_{j} X^{k}-\widetilde{\nabla}_{j} \widetilde{\nabla}_{r} X^{k}=\mathcal{R}_{2}(\varepsilon)_{r j, a}^{k} X^{a}$. Like $\mathcal{R}_{1}(\varepsilon)$, $\mathcal{R}_{2}(\varepsilon) \in \wedge^{2}(T) \otimes T^{*} \otimes T$.

Clearly $\widetilde{\Gamma}[\xi, \eta]-[\widetilde{\Gamma} \xi, \widetilde{\Gamma} \eta] \in \operatorname{Ker}(\pi)$ for $\xi, \eta \in \mathfrak{X}(M)$. The same statement holds for $\widehat{\Gamma}$. Now a straightforward computation using (2), (12) and (21) gives the formulas

$$
\begin{aligned}
(\widetilde{\Gamma}[\xi, \eta]-[\widetilde{\Gamma} \xi, \widetilde{\Gamma} \eta])_{j}^{i} & =\mathcal{R}_{2}(\varepsilon)_{a b, j}^{i} X^{a} Y^{b} \\
(\widehat{\Gamma}[\xi, \eta]-[\widehat{\Gamma} \xi, \widehat{\Gamma} \eta])_{j}^{i} & =\mathcal{R}_{1}(\varepsilon)_{a b, j}^{i} X^{a} Y^{b}=0
\end{aligned}
$$

where $\xi=\left(X^{i}\right), \eta=\left(Y^{i}\right)$.

Now (24) shows that $\widehat{\Gamma}(T) \subset J_{1} T$ is a subalgebroid which is the algebroid of $\varepsilon\left(\mathcal{U}_{0}\right) \subset \mathcal{U}_{1}$. In fact, the splitting $\xi \rightarrow \widehat{\Gamma} \xi$ is the horizontal lift to the principal bundle $\mathcal{U}_{1}^{(p)} \rightarrow M$ which is trivialized by $\varepsilon$. On the other hand, the splitting (12) is conceptually different as we will see in Sections 5,6 . The pair $(\widetilde{\Gamma}, \widehat{\Gamma})$ is a dual pair of affine connections according to [2], [3]. Clearly, $T_{j k}^{i}=0$ implies $\mathcal{R}_{2}(\varepsilon)=\mathcal{R}_{1}(\varepsilon)=0$, but $\mathcal{R}_{2}(\varepsilon)$ need not vanish in general as we will see below.

Finally, using (2) and (20) it is easy to check

$$
\mathcal{L}_{X} \mathcal{L}_{Y} \xi-\mathcal{L}_{Y} \mathcal{L}_{X} \xi=\mathcal{L}_{[X, Y]} \xi
$$

Therefore (25) defines a representation of $J_{1} T$ on $T$. More generally, $J_{k+1} T$ has a representation on $J_{k} T$ (called association in [23], see Definition $8.1 \mathrm{on}$ pg. $362)$ defined by $\mathcal{L}_{X} \xi \stackrel{\text { def }}{=}\left[\pi_{1} X, \xi\right]-i_{\pi_{2} \xi} D(X)$ where $X, \xi$ are sections of $J_{k+1} T \rightarrow$ $M, J_{k} T \rightarrow M$, and $\pi_{1}: J_{k+1} T \rightarrow J_{k} T, \pi_{2}: J_{k+1} T \rightarrow T$ are projections ([23], Lemma 8.32). This association is used in [23] to study deformation cohomology and rigidity as the culmination of this book (pg. 354-393). These concepts are studied also in [8] for a general Lie algebroid $\mathcal{A}$, but it seems to us that the theory introduced in [8] reduces to [23] if $\mathcal{A} \subset J_{k} T$. 


\section{A closed 1-form}

We define

$$
\operatorname{Tr}_{2}(\varepsilon) \stackrel{\text { def }}{=} \mathcal{R}_{2}(\varepsilon)_{r j, a}^{a}=\left[\frac{\partial \Gamma_{a j}^{a}}{\partial x^{r}}\right]_{[r, j]}
$$

The second equality in (26) follows from (22) since $\left[\Gamma_{b r}^{a} \Gamma_{a j}^{b}\right]_{[r, j]}=0$.

We will now define a special 1-form $\omega$ of $J_{1} T$. Recall the algebraic bracket

$$
\{,\}_{p}:\left(J_{1} T\right)_{p} \times\left(J_{1} T\right)_{p} \rightarrow T_{p}
$$

A section $X$ of $J_{1} T$ defines the linear map

$$
\begin{aligned}
X(p): & T_{p} \rightarrow T_{p} \\
: & \xi(p) \rightarrow\{X(p), \widetilde{\Gamma}(\xi(p))\}_{p}
\end{aligned}
$$

We define $\omega$ by

$$
\omega(X) \stackrel{\text { def }}{=} \operatorname{Tr}(X)
$$

Clearly $\omega$ is a 1 -form of $J_{1} T$.

Proposition $6 \omega$ is closed but not locally exact.

Proof: If $X=\left(X^{i}, X_{j}^{i}\right), Y=\left(Y^{i}, Y_{j}^{i}\right)$, then (27) is given by

$$
\{X, Y\}^{i}=X^{a} Y_{a}^{i}-Y^{a} X_{a}^{i}
$$

Thus we get $\{\xi, \widetilde{\Gamma} \eta\}^{i}=X^{a} \Gamma_{a b}^{i} Y^{b}-Y^{a} X_{a}^{i}$ where $\xi=\left(X^{i}\right), \eta=\left(Y^{i}\right)$. So the the linear map (28) is $\left(X^{i}, X_{j}^{i}\right): Y^{i} \rightarrow X^{a} \Gamma_{a b}^{i} Y^{b}-Y^{a} X_{a}^{i}$ and we deduce

$$
\operatorname{Tr}(X)=X^{a} \Gamma_{a b}^{b}-X_{a}^{a}
$$

(4), (29) and (31) show

$$
\omega=\left(\omega^{i}, \omega_{j}^{i}\right)=\left(\Gamma_{i a}^{a},-\delta_{j}^{i}\right)
$$

Substituting (32) into (11), we get

$$
\begin{aligned}
\delta \omega(X, Y) & =X^{a} Y^{c}\left(\frac{\partial \Gamma_{a b}^{b}}{\partial x^{c}}-\frac{\partial \Gamma_{c b}^{b}}{\partial x^{a}}\right) \\
& =\operatorname{Tr}_{1}(\varepsilon)(\pi X, \pi Y) \\
& =0
\end{aligned}
$$

If $\delta f=\omega$ locally, $(32)$ implies $\left(\frac{\partial f}{\partial x^{i}}, 0\right)=\left(\Gamma_{i a}^{a},-\delta_{j}^{i}\right)$, an equality which does not hold for any $f$. 
Proposition 6 shows that the complex computing the cohomology of an algebroid need not be locally exact at the level of 1-forms, which, we believe, is a serious defect from the point of view of characteristic classes since a closed form in such a complex has no interpretation as an obstruction. This fact makes $\omega$ uninteresting from our standpoint and we will use it as a tool for a more relevant construction. For this purpose, note that the algebraic bracket (27) defines the alternating bilinear map

$$
\begin{aligned}
{[,]_{p}: } & T_{p} \times T_{p} \longrightarrow T_{p} \\
: & (\xi(p), \eta(p)) \rightarrow\{\widetilde{\Gamma}(\xi(p)), \widetilde{\Gamma}(\eta(p))\}_{p}
\end{aligned}
$$

We define an ordinary 1-form $w$ on $M$ by

$$
w(\xi) \stackrel{\text { def }}{=} \operatorname{Tr}[\xi, \bullet]
$$

Clearly, $w(\xi)=\omega(\widetilde{\Gamma} \xi)$.

Proposition $7 d w=\operatorname{Tr} \mathcal{R}_{2}(\varepsilon)$

Proof: (35), (34) and (30) give $w(\xi)=\left(\Gamma_{a b}^{b}-\Gamma_{b a}^{b}\right) X^{a}$ or

$$
w_{i}=\Gamma_{i a}^{a}-\Gamma_{a i}^{a}
$$

Using (36), we compute

$$
\begin{aligned}
(d w)_{j i} & =\left[\frac{\partial \Gamma_{i a}^{a}}{\partial x^{j}}-\frac{\partial \Gamma_{a i}^{a}}{\partial x^{j}}\right]_{[j, i]} \\
& =\left[\frac{\partial \Gamma_{a i}^{a}}{\partial x^{j}}\right]_{[j, i]} \quad \text { since }\left[\frac{\partial \Gamma_{i a}^{a}}{\partial x^{j}}\right]_{[j, i]}=\operatorname{Tr}\left(\mathcal{R}_{1}(\varepsilon)\right)_{j i}=0 \\
& =\operatorname{Tr}_{2}(\varepsilon)_{j i} \quad \text { by }(26) \quad \square
\end{aligned}
$$

The derivation of (36) shows that $T_{j k}^{i}(p)=\Gamma_{j k}^{i}(p)-\Gamma_{k j}^{i}(p)$ is a $\operatorname{Hom}\left(T_{p}, T_{p}\right)$ valued 1-form in the present framework: $j$ is the 1 -form index and $i, k$ are the matrix indices. This interpretation of torsion will be of fundamental importance in the construction of secondary characteristic classes in Section 8.

Therefore, $w$ is closed in the deRham complex if and only if $\operatorname{Tr} \mathcal{R}_{2}(\varepsilon)=0$. If we assume $T_{j k}^{i}=0$, then $d w=\operatorname{Tr} \mathcal{R}_{2}(\varepsilon)=\operatorname{Tr} \mathcal{R}_{1}(\varepsilon)=0$. In fact, (19) and (36) show that $T_{j k}^{i}=0$ implies $w=0$ ! The condition $T_{j k}^{i}=0$ is very strong: If $M$ is compact, it forces $M$ to be of the form $G / \Delta$ where $G$ is the affine linear group $A L(n, \mathbb{R})$ and $\Delta \subset G$ is a discrete subgroup (see [16], Theorem 4.2). However, our assumption in the next section will allow $G$ to be any connected Lie group. 


\section{From Lie algebroid to Lie algebra}

Proposition 8 The following are equivalent:

i) $\mathcal{R}_{2}(\varepsilon)=0$

ii) $\widetilde{\Gamma}(T) \subset J_{1} T$ is a subalgebroid

iii) $\left[\mathfrak{X}_{\varepsilon}(M), \mathfrak{X}_{\varepsilon}(M)\right] \subset \mathfrak{X}_{\varepsilon}(M)$

Proof: The equivalence $i) \Leftrightarrow i i$ ) follows from (23).

iii $) \Rightarrow i)$ : Let $\xi, \eta \in \mathfrak{X}_{\varepsilon}(M)$. We have $j_{1}[\vartheta, \gamma]=\left[j_{1} \vartheta, j_{1} \gamma\right]$ for any $\vartheta, \gamma \in$ $\mathfrak{X}(M)$ by $(3)$. By Lemma $5, j_{1} \vartheta=\widetilde{\Gamma}(\vartheta)$ for any $\vartheta \in \mathfrak{X}_{\varepsilon}(M)$ and therefore $j_{1}[\xi, \eta]=\widetilde{\Gamma}\left([\xi, \eta]\right.$ since $[\xi, \eta] \in \mathfrak{X}_{\varepsilon}(M)$. So we deduce $\widetilde{\Gamma}([\xi, \eta])=[\widetilde{\Gamma} \xi, \widetilde{\Gamma} \eta] \quad \xi, \eta \in$ $\mathfrak{X}_{\varepsilon}(M)$. If $\left(\xi_{i}\right)$ is a basis for $\mathfrak{X}_{\varepsilon}(M)$, any $\vartheta \in \mathfrak{X}(M)$ can be written as $\vartheta=f^{i} \xi_{i}$ for some functions $f^{i}$ on $M$ and the conclusion follows since $\mathcal{R}_{2}(\varepsilon)(\vartheta, \gamma)$ is linear in its arguments $\vartheta, \gamma$.

$i) \Rightarrow$ iii) : Let $\xi, \eta \in \mathfrak{X}_{\varepsilon}(M)$. (3), (23) and Lemma 5 give $j_{1}[\xi, \eta]=\widetilde{\Gamma}([\xi, \eta])$. Therefore $[\xi, \eta]$ is a solution of (14) and the conclusion follows from Lemma 5

We will denote the conditions of Proposition 8 by A. Propositions 7, 8 now give

Corollary 9 A implies $d w=0$

If $\xi_{(i)}$ is a basis of $\mathfrak{X}_{\varepsilon}(M)$, then $\left[\xi_{(i)}, \xi_{(j)}\right]^{k}=c_{i j}^{a} \xi_{(a)}^{k}$ for some functions $c_{i j}^{a}$ on $M$. These functions are constant if and only if $i$ ii) of Proposition 8 holds. We now make the following

Definition 10 If a differentiable manifold $M$ admits a splitting $\varepsilon$ such that $\mathbf{A}$ holds, then $M$ together with $\varepsilon$ is called a local Lie group.

Our definition of local Lie group coincides with the one given in [20] in view of Theorem 18 in [20] and Proposition 8. For a local Lie group $M$, we will construct in Section 7 a Lie group $\widetilde{G}$ (see the proof of Proposition 15) whose Lie algebra can be identified with $\mathfrak{X}_{\varepsilon}(M)$.

Henceforth in this section we assume that $M$ is a local Lie group. Therefore $[w] \in H_{d R}^{1}(M, \mathbb{R})$. Recalling the formula $w(\xi)=\omega(\widetilde{\Gamma} \xi)$, we may identify $w$ with the restriction of $\omega$ to $\widetilde{\Gamma}(T)$. Unlike $\omega, w$ is clearly locally exact and can be also globally exact in the deRham complex. Our main concern in Section 7 will be the following question

Q : What is the obstruction to the exactness of $w$ ?

We now define the evaluation map

$$
\begin{aligned}
e_{p} & : \mathfrak{X}_{\varepsilon}(M) \longrightarrow T_{p} \\
& : \xi \longrightarrow \xi(p)
\end{aligned}
$$

where $p \in M$ is arbitrary. Recalling the alternating bilinear map $[,]_{p}$ defined by (34), we have 
Proposition 11 e preserves brackets.

Proof: This is a straightforward verification using (9) and (34).

Proposition 11 shows that the Lie algebra structure of $\mathfrak{X}_{\varepsilon}(M)$ is determined at any point $p \in M$ in terms of $[,]_{p}$. The Jacobi identity imposes now restrictions on the components $\Gamma_{j k}^{i}(p)$ which we do not write down explicitly as we do not use them. It is not difficult to check that we now have $\left(\varepsilon^{p, q}\right)_{*}[\xi, \eta]_{p}=$ $\left[\left(\varepsilon^{p, q}\right)_{*} \xi,\left(\varepsilon^{p, q}\right)_{*} \eta\right]_{q}$ and $w$ is $\varepsilon$-invariant: $w_{p}(\xi(p))=w_{q}\left(\left(\varepsilon^{p, q}\right)_{*} \xi(p)\right)$.

\section{$7 \quad\left[w_{1}\right]$ as obstruction to globalizability}

When is a manifold a Lie group? This question is quite old and studied by several authors, for instance, see [12], [13], [26] for proofs based on the formalism of Maurer-Cartan forms. A different proof is outlined in [2] which is more in the spirit of this paper: assuming $\mathbf{A}$, the dual pair of flat affine connections $(\widetilde{\Gamma}, \widehat{\Gamma})$ imposes a local Lie group structure on $M$ by Theorem A in [2]. We will give a slightly different proof of this fact in this section which unifies the dual pair $(\widetilde{\Gamma}, \widehat{\Gamma})$ as the single object $\varepsilon$ and the curvatures $\mathcal{R}_{1}(\varepsilon), \mathcal{R}_{2}(\varepsilon)$ as $\mathcal{R}(\varepsilon)$ which is the curvature of $\varepsilon$ from our standpoint. Our proof will also interpret the obstruction $[w]$ to globalizability of a local Lie group as a secondary characteristic class in view of Corollary 9 and Proposition 7.

By the definition of a 1-arrow, for any $p \in\left(U, x^{i}\right)$ and $q \in\left(V, y^{i}\right)$, there exists a local diffeomorphism $f$ with $f(p)=q$ satisfying

$$
\frac{\partial f^{i}}{\partial x^{j}}(p)=\varepsilon_{j}^{i}(p, f(p))
$$

Thus the splitting $\varepsilon$ determines a global $P D E$ on $\mathcal{U}_{0}=M \times M$ which is locally given by (39). Note that $f$ in (39) depends on the point $(p, q)$ and we may not be able to find some $f$ which works for all $p \in U$ as the $P D E$ (39) may not admit any local solutions. The integrability condition of (39) is given by

$$
\left[\frac{\partial \varepsilon_{j}^{i}(x, y)}{\partial x^{k}}+\frac{\partial \varepsilon_{j}^{i}(x, y)}{\partial y^{a}} \varepsilon_{k}^{a}(x, y)\right]_{[k j]} \stackrel{\text { def }}{=} \mathcal{R}(\varepsilon)_{k j}^{i}(x, y)=0
$$

If (39) admits a solution $f$ with $f(p)=q$ for any $(p, q) \in U \times V$, then clearly $\mathcal{R}(\varepsilon)_{k j}^{i}(x, y)=0$ on $U \times V$. Conversely, by the well known existence and uniqueness theorem for first order systems of $P D E$ 's, if $\mathcal{R}(\varepsilon)_{k j}^{i}(x, y)=0$ on $U \times V$, then we may assign any 1-arrow in $\varepsilon\left(\mathcal{U}_{0}\right)$ with source at $p \in U$ and target at $q \in V$ as initial condition and solve (39) uniquely for some $f$ on $\bar{U} \subset U$ satisfying $f(p)=q$.

Definition $12 \varepsilon$ is flat if for any $p, q \in M$, there exist neighborhoods $p \in$ $\left(U, x^{i}\right), q \in\left(V, y^{i}\right)$ such that $\mathcal{R}(\varepsilon)(x, y)=0$ on $U \times V$.

It is easy to check that $\mathcal{R}(\varepsilon)(p, q) \in \Lambda^{2}\left(T_{p}^{*}\right) \otimes T_{q}$. In particular, flatness has a meaning independent of coordinates. Note that $\mathcal{R}(\varepsilon)$ is defined on $M \times M$ 
and not on $M$. Also, $\mathcal{R}(\varepsilon)(p, p)=0$ since identity diffeomorphism is the unique solution of (39). So $\mathcal{R}(\varepsilon)$ vanishes identically on the diagonal of $M \times M$.

Now suppose $\varepsilon$ is flat. Since $\varepsilon$ preserves the composition and inversion of 1-arrows, the local diffeomorphisms which integrate these 1-arrows form a pseudogroup with the property that any local diffeomorphism of this pseudogroup is determined on its domain by any of its 1-arrows. We denote this pseudugroup by $\mathcal{S}_{\varepsilon}$. Clearly, if $f \in \mathcal{S}_{\varepsilon}$, then all 1-arrows of $f$ belong to $\varepsilon\left(\mathcal{U}_{0}\right)$ by (39).

Now let $f_{1} \in \mathcal{S}_{\varepsilon}$ with domain $U_{1}$ and $\alpha(t), 0 \leq t \leq 1$ a (continuous) path in $M$ with $\alpha(0) \in U_{1}$. Suppose there exist open sets $U_{i}, 1 \leq i \leq k$, which cover the image of $\alpha$ and $f_{i} \in \mathcal{S}_{\varepsilon}$ with domain $U_{i}$ such that $f_{i}=f_{j}$ on $U_{i} \cap U_{j}$. We will call this data a continuation of $f_{1}$ along $\alpha$. The continuation of $f \in \mathcal{S}_{\varepsilon}$ along $\alpha$ is unique with the obvious meaning of uniqueness. Since $f \in \mathcal{S}_{\varepsilon}$ is determined by its 1 -arrows, continuing $f$ is the same as continuing its 1 -arrow with source at $\alpha(0)$.

Definition 13 Suppose $\varepsilon$ is flat. If all elements of $\mathcal{S}_{\varepsilon}$ can be continued along paths, then $\varepsilon$ is complete.

Lemma 14 If $M$ is compact, then $\varepsilon$ is complete.

Proof: Let $f \in \mathcal{S}_{\varepsilon}$ with domain $U$ and $\alpha(t)$ be a path with $\alpha(0)=p \in U$. Let $f(p)=q$ and $f^{p, q}$ denote the 1-arrow of $f$. Therefore $f^{p, q}$ has a (unique) continuation $f^{\alpha(t), f(\alpha(t))}$ for small $t$. Let $t_{0} \stackrel{\text { def }}{=} \sup \left\{t \in(0,1] \mid f^{p, q}\right.$ has a continuation on $(0, t)\}$. Let $\beta(t)$ denote the curve traversed by the targets of the 1 -arrows as their source is continued along $\alpha$. Since $\beta$ is locally the image of $\alpha$ by a local diffeomorphism belonging to $\mathcal{S}_{\varepsilon}, \beta$ is continuous. Letting $t_{n} \rightarrow t_{0}^{-}$, $\beta\left(t_{n_{k}}\right) \rightarrow r \in M$ since $M$ is compact. Since $\beta$ is continuous, we conclude $\beta(t) \rightarrow r$ as $t \rightarrow t_{0}^{-}$. Thus we obtain a 1-arrow $g^{\alpha\left(t_{0}\right), r}$ which belongs to $\varepsilon\left(\mathcal{U}_{0}\right)$ since $\varepsilon\left(\mathcal{U}_{0}\right) \subset \mathcal{U}_{1}$ is closed. Since $\mathcal{R}(\varepsilon)=0$, we can assign $g^{\alpha\left(t_{0}\right), r}$ as initial condition and solve (39) uniquely for some $g \in \mathcal{S}_{\varepsilon}$ such that the 1-arrow of $g$ is $g^{\alpha\left(t_{0}\right), r}$ which will give a contradiction unless $t_{0}=1$.

Now let $\widetilde{G}$ be any connected Lie group and $\Delta \subset \widetilde{G}$ a discrete subgroup. We call the (left) coset space $\widetilde{G} / \Delta$ a discrete quotient.

The geometric meaning of flatness is clarified by the following

Proposition 15 If $M$ is a discrete quotient, then $M$ posesses a canonical flat $\varepsilon$. Conversely, let $\varepsilon$ be a flat splitting. If $\varepsilon$ is complete, then $M$ is a discrete quotient.

Proof: For the first statement, let $\pi: \widetilde{G} \rightarrow \widetilde{G} / \Delta=M$ be the covering map. It is well known that $M$ is parallelizable. In fact, let $p, q \in M$. Since the action of $\Delta$ on $\widetilde{G}$ commutes with $\pi$, any 1-arrow on $\widetilde{G}$ (induced by the action of some $g \in \widetilde{G})$ with source in $\pi^{-1}(p)$ and target in $\pi^{-1}(q)$ projects to the same 1-arrow on $M$ from $p$ to $q$. So we get a splitting $\varepsilon$ on $M$. Now $\mathcal{R}(\varepsilon)=0$ because the action of $\widetilde{G}$ on itself projects locally to $M$ giving a pseudogroup on $M$ whose local diffeomorphisms integrate these 1-arrows on $M$ (clearly, this pseudugroup is $\mathcal{S}_{\varepsilon}$ ). 
For the converse, let $\pi: \widetilde{M} \rightarrow M$ be the universal covering map. $\mathcal{S}_{\varepsilon}$ pulls back to a pseudogroup $\widetilde{\mathcal{S}}_{\varepsilon}$ on $\widetilde{M}$ with the same property: any $\widetilde{f} \in \widetilde{\mathcal{S}}_{\varepsilon}$ is determined on its domain by any of its 1 -arrows. Let $\widetilde{f} \in \widetilde{\mathcal{S}}_{\varepsilon}$ and let $\widetilde{\alpha}(t), 0 \leq t \leq 1$, be a path with $\widetilde{\alpha}(0) \in \operatorname{Dom}(\widetilde{f})$. Now $\widetilde{f}$ and $\widetilde{\alpha}(t)$ project to $f \in \mathcal{S}_{\varepsilon}$ and $\alpha(t)$ (restricting $\operatorname{Dom}(\widetilde{f})$ if necessary) and we can translate $f$ along $\alpha$ by Definition 13, obtaining some $g \in \mathcal{S}_{\varepsilon}$ with $\alpha(1) \in \operatorname{Dom}(g)$. Lifting this "analytic continuation" to $\widetilde{M}$, we obtain some $\widetilde{g} \in \widetilde{\mathcal{S}}_{\varepsilon}$ with $\widetilde{\alpha}(1) \in \operatorname{Dom}(\widetilde{g})$. Since $\widetilde{M}$ is simply connected, the standard monodromy argument shows that this analytic continuation is independent of the choice of the path from $\widetilde{\alpha}(0)$ to $\widetilde{\alpha}(1)$. Therefore, any $\widetilde{f} \in \widetilde{\mathcal{S}}_{\varepsilon}$ extends uniquely to a global diffeomorphism $\widetilde{f}^{e}$ on $\widetilde{M}$. We define $\widetilde{G} \stackrel{\text { def }}{=}\left\{\widetilde{f^{e}} \mid \widetilde{f} \in \widetilde{\mathcal{S}}\right\}$ and check that $\widetilde{G}$ is a group. Clearly $\widetilde{G}$ acts simply transitively on $\widetilde{M}$. In fact, it is not difficult to show that $\widetilde{G}$ is a Lie group so that $\widetilde{M}$ is the underlying manifold of $\widetilde{G}$. Letting $\Delta=$ (deck transformations on $\widetilde{M}) \simeq \pi_{1}(M)$, we get $\widetilde{G} / \Delta=M$ and $M$ is a discrete quotient.

The next proposition will add another equivalent condition to the conditions of Proposition 8 which we denoted by $\mathbf{A}$.

Proposition $16 \mathcal{R}(\varepsilon)=0$ if and only if $\mathbf{A}$ holds.

Proof: Suppose $\mathcal{R}(\varepsilon)=0$ on $M \times M$. We differentiate the LHS of (40) with respect to $y$ and set $y=x$, which gives $\mathcal{R}_{2}(\varepsilon)=0$ after some computation using (11). For the converse, we first observe that $\mathcal{R}(\varepsilon)=0$ on $M \times M$ if and only if $\mathcal{R}(\varepsilon)=0$ on some neighboorhood $U \times U$ of $(p, p)$ for any $p \in M$. To see this, let $p, q \in M$ and consider the 1 -arrow $\varepsilon^{p, q}$. We choose any path from $p$ to $q$ and take a covering $\left(U_{i}\right)$ of this path such that $\mathcal{R}(\varepsilon)=0$ on $U_{i} \times U_{i}$. We subdivide this path into small paths such that each small path is contained in some $U_{i}$. Let $p_{k}, p_{k+1}$ be the initial and terminal points of the $k^{\prime}$ th small path. The composition of the 1-arrows $\varepsilon^{p_{k}, p_{k+1}}$ is $\varepsilon^{p, q}$ and each 1-arrow $\varepsilon^{p_{k}, p_{k+1}}$ integrates by assumption to a local diffeomorphism which is a solution of (39). Now the composition of these local diffeomorphisms integrates $\varepsilon^{p, q}$ and therefore is a solution of (39) which shows $\mathcal{R}(\varepsilon)(p, q)=0$, proving the claim.

Now suppose $\mathcal{R}_{2}(\varepsilon)=0$ and let $\left(U, x^{i}\right)$ be any coordinate patch. We choose some $p \in U$, a basis of the tangent space at $p$ and extend this basis to $\varepsilon$-invariant vector fields $\xi_{(i)}$ on $U, 1 \leq i \leq n$. Now $\left[\xi_{(i)}(x), \xi_{(j)}(x)\right]=c_{i j}^{a}(x) \xi_{(a)}(x)$ for some functions $c_{i j}^{a}(x)$ on $U$. Since $\mathcal{R}_{2}(\varepsilon)=0$, the functions $c_{i j}^{a}(x)$ are constant by Proposition 8. By Lie's Third Fundamental Theorem (LTFT), there exists a local Lie group acting on $U$ (shrinking $U$ if necessary) with infinitesimal generators $\xi_{(i)}$. Now the proof of $L T F T$ (see [25], pg. 398-415) shows that the 1 -arrows of the local diffeomorphisms induced by this local Lie group action coincide with the 1 -arrows of $\varepsilon\left(\mathcal{U}_{0}\right)$. Thus $\mathcal{R}(\varepsilon)=0$ on $U \times U$ and therefore on $M \times M$ since $U$ is arbitrary.

In view of Propositions 8,16 , a local Lie group is a differentiable manifold $M$ together with a flat splitting $\varepsilon$. We do not know whether a parallelizable manifold $M$ can be a local Lie group in different ways, that is, whether it can admit two flat splittings $\varepsilon, \bar{\varepsilon}$ with nonisomorphic Lie algebras $\mathfrak{X}_{\varepsilon}(M), \mathfrak{X}_{\bar{\varepsilon}}(M)$. 
Henceforth, the statement " $M$ is a local Lie group" will always refer to $M$ together with its flat splitting which we fix once and for all.

Proposition 8 shows that the condition $\mathcal{R}_{2}(\varepsilon)=0$ is equivalent to the hypothesis of LTFT and the proof of Proposition 16 shows that $\mathcal{R}(\varepsilon)=0$ is equivalent to its conclusion. Therefore, the program outlined in the Introduction, if viewed locally, is nothing but a natural generalization of of LTFT from simply transitive actions (where the geometric order $m=0$ ) to transitive and effective actions (where $m$ is arbitrary).

As we see from the proof of Proposition $16, \widetilde{\mathcal{S}}_{\varepsilon}$ globalizes to the Lie group $\widetilde{G}$ with underlying manifold $\widetilde{M}$. However, $\mathcal{S}_{\varepsilon}$ may globalize to a Lie group $G$ already on $M$, that is, $\varepsilon$ may have "trivial monodromy" already on $M$ (on the other hand, recall that $\varepsilon$, as the trivialization of the principal bundle $\mathcal{U}_{1}^{(p)} \rightarrow M$, has necessarily trivial monodromy).

Definition 17 A local Lie group $M$ is globalizable if any $f \in \mathcal{S}_{\varepsilon}$ extends (necessarily uniquely) to a global diffeomorphism of $M$.

So $M$ is globalizable if and only if there are no obstructions to the existence of global solutions of (39) in which case the global diffeomorphisms of $\mathcal{S}_{\varepsilon}$ act simply transitively on $M$ and have the structure of a Lie group $G$. In this case, we simply say that $M$ is a Lie group. Observe that the statement " $M$ is a Lie group" refers to two different objects: the pair $(G, M)$ where $G$ is a transformation group of $M$ which acts simply transitively and the abstract Lie group $G$. We can identify $G$ and $M$ by choosing some point $e \in M$ and map $g \in G$ to its 0 -arrow from $e$ to $g(e)$. Clearly, this map $G \rightarrow M$ is a bijection, in fact, a diffeomorphism, but there is no canonical identification. We will continue to make such identification as we did already in the proof of Proposition 15.

Definition 17 of globalizability coincides with the one given in [20] if $M$ is complete.

Using the notation and setting of Proposition 15, the next proposition characterizes globalizability of $M$ in terms of the discrete subgroup $\Delta \subset \widetilde{G}$.

Proposition $18 M$ is a Lie group if and only if $\Delta \subset \widetilde{G}$ is a normal (hence central) subgroup.

Proof: If $\Delta \subset \widetilde{G}$ is a normal subgroup, then $\pi: \widetilde{G} \longrightarrow \widetilde{G} / \Delta=M$ is a homomorphism of Lie groups and surely $M$ is globalizable.

For the converse, suppose that $M$ is globalizable. Let $\pi(e)=x, g \in \widetilde{G}$, $\pi(g)=y$. Clearly $\pi(d(0))=x$ for all $d(0) \in \Delta$. Now the action of $g$ on $\widetilde{G}$ defines a local diffeomorphism which maps a neighborhood of the identity $e$ to a neighborhood of $g$. This local diffeomorphism projects to some $f(1) \in \mathcal{S}_{\varepsilon}$ with $f(1)(x)=y$. We choose a path $A(s), 0 \leq s \leq 1$ with $A(0)=e, A(1)=d(0)$ and consider the loop $\pi \circ A=\bar{A}$ at $x$. We now continue $f(1)$ along the loop $\bar{A}$ turning the source $x$ to its initial value. Thus the target $f(1)(x)=y$ traces a curve starting from $y$. Since $f(1)$ globalizes by assumption, $y$ also turns to its initial value and this curve is also a loop at $y$. We lift this loop to a curve $B(s)$, 
$0 \leq s \leq 1$, which starts from $B(0)=g$. Since $\pi(B(1))=\pi(B(0))=y$, there exists a unique $d(1) \in \Delta$ with $B(1)=d(1) g$. We now lift the continuation of $f(1) \in \mathcal{S}_{\varepsilon}$ to $\widetilde{G}$ as in the proof of Proposition 15. Since the action of $g$ is a global diffeomorphism and $g$ remains the same during this continuation, we conclude $g A(s)=B(s)$ for all $s$. Therefore $g d(0)=g A(1)=g B(1)=d(1) g$. We claim that $d(0)=d(1)$. This will finish the proof since $g$ and $d(0)$ are both arbitrary in $g d(0)=d(0) g$.

The prove the claim, we choose a path $g(t)$ with $g(0)=e, g(1)=g$. Replacing $g=g(1), f(1) \in \mathcal{S}_{\varepsilon}$ with $g(t), f\left(\pi(g(t)) \in \mathcal{S}_{\varepsilon}\right.$ and repeating the above argument, we obtain a loop at $\pi(g(t)$ which lifts to a curve $H(s, t), 0 \leq s \leq 1$ with $H(0, t)=g(t)$. Clearly, $H(s, 0)=A(s)$ and $H(s, 1)=B(s)$. By construction, $H(s, t)$ is continuous on $[0,1] \times[0,1]$ and therefore $H(1, t)$ is a curve from $d(0)$ to $d(1) g$. Since $\pi(H(0, t))=\pi(H(1, t))$, there exists a unique $d(t) \in \Delta$ satisfying $d(t) g(t)=H(1, t)$. We define $F(t) \stackrel{\text { def }}{=} H(1, t) g(t)^{-1} \in \Delta$ for all $t$. Since $\Delta$ is discrete and $F(t)$ is continuous, $F(t)$ is constant which proves the claim.

Recalling Definition 10, a Lie group is clearly a complete local Lie group. The proof of the next corollary is immediate from the proof of Proposition 15.

Corollary 19 A simply connected and complete local Lie group is a Lie group.

Henceforth in this section, we assume that $M$ is a complete local Lie group and therefore a discrete quotient in view of Proposition 16.

Now, since the 1-form $w$ is closed by Corollary 9, it is locally exact and our aim is to find an explicit local primitive of $w$. For this purpose, we fix some $e \in M$ and a simply connected coordinate patch $e \in\left(U, x^{i}\right)$. For $a, b \in U$, we define $a b \in M$ as follows: since $\mathcal{R}(\varepsilon)=0$, the 1 -arrows $\varepsilon^{e, a}$ and $\varepsilon^{e, b}$ integrate to local diffeomorphisms $f, g \in \mathcal{S}_{\varepsilon}$ with $f(e)=a$ and $g(e)=b$ and $f, g$ are both defined on $U$ since $U$ is simply connected and $\varepsilon$ is complete. We define

$$
a b \stackrel{\text { def }}{=}(f \circ g)(e)=f(b) \in M
$$

In (41) we adhere to the standard convention of composing from right to left. Note that (41) depends on our choice of the base point $e$ and $a b$ may not be in $U$ unless $a, b$ are sufficiently close to $e$. At this point, it is worthwhile to observe that the classical concept of local Lie group is modelled on this local structure, that is, the set $U$ together with the local multiplication (41), whereas a local Lie group $M$ according to Definition 10 is essentially a global concept. In particular, it may be misleading to imagine a local Lie group $M$ as an open neighborhood of identity in some global Lie group $G$. In fact, the reader may have observed that it is possible to define a Lie group as a globalizable local Lie group.

The local multiplication (41) determines the local left and right translations $L_{a}, R_{a}$ defined by $L_{a}(x)=a x$ and $R_{a}(x)=x a$ where $a, x \in U$. Thus we may consider the 1-arrows of $L_{a}$ and $R_{a}$ with source at $e$ and target at $a$, that is $j_{1}\left(L_{a}\right)^{e, a}$ and $j_{1}\left(R_{a}\right)^{e, a}$ which we want to compute now in coordinates. 
Let $f \in \mathcal{S}_{\varepsilon}, f(e)=a$ and $f$ be defined on $U$. Also, for any $x \in U$, let $g_{x} \in \mathcal{S}_{\varepsilon}$ such that the diffeomorphism $y \rightarrow g_{x}(y)$ is defined on $U$ and is the unique solution of (39) in the variable $y$ satisfying the initial condition $g_{x}(e)=x$. We also have $g_{e}(y)=y$ since the only solution of (39) which fixes $e$ is identity on $U$. Now (41) gives $L_{a}(x)=a x=\left(f \circ g_{x}\right)(e)=f(x)$ and therefore

$$
\left[j_{1}\left(L_{a}\right)^{e, a}\right]_{j}^{i}=\left[\frac{\partial f^{i}(x)}{\partial x^{j}}\right]_{x=e}=\varepsilon_{j}^{i}(e, a)
$$

We see from (42) that the assumption $\mathbf{A}$ is not needed to define $j_{1}\left(L_{a}\right)^{e, a}$ and the "local left 1-arrows" are actually global as they coincide with the 1-arrows of $\varepsilon$.

Similarly

$$
\left[j_{1}\left(R_{a}\right)^{e, a}\right]_{j}^{i}=\left[\frac{\partial\left(\left(g_{x} \circ f\right)(e)\right)^{i}}{\partial x^{j}}\right]_{x=e}=\left[\frac{\partial g_{x}^{i}(a)}{\partial x^{j}}\right]_{x=e}
$$

We see from (43) that the definition of $j_{1}\left(R_{a}\right)^{e, a}$ depends essentially on $\mathbf{A}$ and "right 1-arrows" are local and defined only on $U$. The following points are worth mentioning: if $a \in M, b \in U$, then $a b$ is still defined by (41), whereas to define $b a$ we must continue the domain of $f$ so that it contains the point $b$, but continuations along different paths may give different values for $f(b)$ and it may be impossible to define $b a$ uniquely. Equivalently, we have the multiplication map $M \times U \rightarrow M$ but not necessarily $U \times M \rightarrow M$, in contrast to the classical definition of a local Lie group. It is due to this fact that left 1-arrows are global whereas right 1-arrows are not. Also, left and right are reversed if we change our convention of composition from right to left in (41), which shows that left and right are nonconcepts for the local Lie group $M$ (even if it is globalizable!) whereas they are honest concepts for the Lie group $G$.

We now define the function $A d_{e}: U \rightarrow \mathcal{U}_{1}^{e, e}$ by

$$
A d_{e}(a) \stackrel{\text { def }}{=}\left[j_{1}\left(L_{a}\right)^{e, a}\right]^{-1} \circ j_{1}\left(R_{a}\right)^{e, a}
$$

We call $A d_{e}$ the local adjoint map based at $e$. Using (42) and (43) we get

$$
\left[A d_{e}(a)\right]_{j}^{i}=\varepsilon_{b}^{i}(a, e)\left[\frac{\partial g_{x}^{b}(a)}{\partial x^{j}}\right]_{x=e}
$$

Since $A d_{e}(e)=i d^{e, e}, \operatorname{det}\left(A d_{e}(x)\right)$ is positive at $x=e$ and therefore positive on $U$ since it is nonzero and continuous.

Lemma 20 Let $A(x)=\left[\alpha_{j}^{i}(x)\right]$ be an $n \times n$ matrix whose entries are smooth functions on an open set $U \subset \mathbb{R}^{n}$. Suppose that $A(x)$ is invertible on $U$ with inverse $B(x)=\left[\beta_{j}^{i}(x)\right]$. Then

$$
(\operatorname{det} A)^{-1} \frac{\partial \operatorname{det}(A)}{\partial x^{j}}=\frac{\partial \alpha_{b}^{c}}{\partial x^{j}} \beta_{c}^{b}
$$


Proof: (46) follows easily from the cofactor expansion of $\operatorname{det}(A)$ (see [25], pg. 8, formula 7.2).

Note that the $R H S$ of (46) is $\operatorname{Tr}\left(\frac{\partial A}{\partial x^{j}} A^{-1}\right)$. If $\operatorname{det}(A)$ is positive on $U$, then the $L H S$ of (46) is equal to

$$
\frac{\partial \log (\operatorname{det}(A))}{\partial x^{j}}
$$

Proposition $21-d\left(\log \left(\operatorname{det} A d_{e}\right)\right)=w$ on $U$.

Proof: We first show that $-d\left(\log \left(\operatorname{det} A d_{e}\right)\right)$ and $w$ have the same values at $x=e$. Using (45), Lemma 20, (11) and (36) we compute

$$
\begin{aligned}
-\left[d\left(\log \left(\operatorname{det} A d_{e}\right)\right)(e)\right]_{i} & =-\left[\frac{\partial}{\partial y^{i}} \log \operatorname{det}\left(\varepsilon_{b}^{j}(y, e) \frac{\partial g_{x}^{b}(y)}{\partial x^{k}}\right)\right]_{x=e, y=e} \\
& =-\left[\frac{\partial}{\partial y^{i}}\left(\varepsilon_{b}^{c}(y, e) \frac{\partial g_{x}^{b}(y)}{\partial x^{d}}\right)\right]_{x=e, y=e}\left(\delta_{c}^{d}\right) \\
& =-\left[\frac{\partial}{\partial y^{i}}\left(\varepsilon_{b}^{c}(y, e) \frac{\partial g_{x}^{b}(y)}{\partial x^{c}}\right)\right]_{x=e, y=e} \\
& =-\left[\frac{\partial \varepsilon_{b}^{c}(y, e)}{\partial y^{i}} \frac{\partial g_{x}^{b}(y)}{\partial x^{c}}+\varepsilon_{b}^{c}(y, e) \frac{\partial^{2} g_{x}^{b}(y)}{\partial y^{i} \partial x^{c}}\right]_{x=e, y=e} \\
& =-\left[\frac{\partial \varepsilon_{b}^{c}(y, e)}{\partial y^{i}}\right]_{y=e}\left(\delta_{c}^{b}\right)-\left(\delta_{b}^{c}\right) \frac{\partial}{\partial x^{c}}\left[\frac{\partial g_{x}^{b}(y)}{\partial y^{i}}\right]_{x=e, y=e} \\
& =\Gamma_{i b}^{b}(e)-\frac{\partial}{\partial x^{b}}\left[\frac{\partial g_{x}^{b}(y)}{\partial y^{i}}\right]_{x=e, y=e} \\
& =\Gamma_{i b}^{b}(e)-\left[\frac{\partial \varepsilon_{i}^{b}(e, x)}{\partial x^{b}}\right]_{x=e} \\
& =\Gamma_{i b}^{b}(e)-\Gamma_{b i}^{b}(e) \\
& =[w(e)]_{i}
\end{aligned}
$$

which proves the claim. Now let $\bar{e} \in U$ another base point. It is easy to check that

$$
\log \left(\operatorname{det} A d_{e}(x)\right)=\log \left(\operatorname{det} A d_{\bar{e}}(x)\right)+\log \left(\operatorname{det} A d_{e}(\bar{e})\right)
$$

Differentiating (48) at $x=\bar{e}$ and using (47), we get

$$
-d\left(\log \left(\operatorname{det} A d_{e}\right)(\bar{e})=w(\bar{e})\right.
$$

which finishes the proof since $\bar{e}$ is arbitrary.

Note that (44) defines $A d$ by the formula $x \rightarrow g^{-1} x g$ rather than the standard one $x \rightarrow g x g^{-1}$ which accounts for the minus sign in Proposition 21. The reason for our choice is that it was easier to invert the local formula for $j_{1}\left(L_{a}\right)^{e, a}$. 
Now let $M$ be a Lie group, that is, $M$ be globalizable. We fix some $e \in M$ and define the function $A d: M \rightarrow \mathcal{U}_{1}^{e, e}$ by (44). Observe that $A d$ is now defined on $M$ since right 1-arrows are globally defined since $M$ is globalizable. Thus we have

Proposition $22 w$ is exact on a Lie group $M$ with primitive $-d\left(\log \left(\operatorname{det} A d_{e}\right)\right)$.

We refer the reader to [15], Propositions 6.29, 6.34 for an intriguing relation of the primitive $-\log \left(\operatorname{det} A d_{e}\right)$ to the Maslov index. See also [9] for another interpretation of $A d_{e}$ in $-\log \left(\operatorname{det} A d_{e}\right)$ as a Nijenhuis tensor.

Proposition 23 Let $f: M \rightarrow N$ be a smooth covering map. If $N$ is a local Lie group, then $f$ determines a local Lie group structure on $M$ and $f^{*} w_{N}=w_{M}$.

Proof: Straightforward using definitions.

Proposition 24 Let $\widetilde{G}$ be a connected Lie group, $\Delta \subset \widetilde{G}$ a discrete subgroup and $\pi: \widetilde{G} \rightarrow \widetilde{G} / \Delta=M$ the covering map. Then $w_{M}$ is exact if and only if $\log \left(\operatorname{det}\left(A d_{\widetilde{G}}\right)\right)(a x)=x, a \in \Delta, x \in \widetilde{G}$, that is, the function $\log \left(\operatorname{det}\left(A d_{\widetilde{G}}\right)\right)$ is automorphic with respect to $\Delta$.

Proof: $\log \left(\operatorname{det}\left(A d_{\widetilde{G}}\right)\right)$ is constant on the orbits of $\Delta$ if and only if there exists a function $h$ on $M=\widetilde{G} / \Delta$ with $h \circ \pi=\log \left(\operatorname{det}\left(A d_{\widetilde{G}}\right)\right)$. We choose an open set $U \subset H$ such that $\pi: U \rightarrow V \subset M$ is a diffeomorphism. Now $w_{\widetilde{G}}=$ $d \log \left(\operatorname{det}\left(A d_{\widetilde{G}}\right)\right)=d(h \circ \pi)=\pi^{*}(d h)$ on $U$. Therefore $\left(\pi^{*}\right)^{-1}\left(w_{\widetilde{G}}\right)=w_{M}=d h$ on $V$ by Lemma 23. Since $V$ is arbitrary, we conclude $d h=w_{M}$ on $M$ and the conclusion follows.

Recall that a connected Lie group is called unimodular if $\operatorname{det}(A d)=1$. $G$ is unimodular if and only if it admits a nonzero invariant $n$-form.

Corollary 25 If $M$ is the discrete quotient of a connected and unimodular Lie group $\widetilde{G}$, then $w_{M}=0$.

Proof: By Lemma 23 and Proposition 22, we have $\pi^{*} w_{M}=w_{\widetilde{G}}=d \log (\operatorname{det} A d)=$ 0 . Therefore $w_{M}=0$ since $\pi$ is a local diffeomorphism.

Many important Lie groups are unimodular: abelian, nilpotent, compact, semisimple and reductive. Now let $B \subset S L(2, \mathbb{R})$ the Borel subgroup of upper triangular matrices. An easy computation $\operatorname{shows} \operatorname{det}\left(a d\left[\begin{array}{ll}a & b \\ 0 & c\end{array}\right]\right)=\frac{c}{a}$. Let $\Delta \subset B$ be the subgroup of matrices of the form $\left[\begin{array}{cc}2^{n} & 0 \\ 0 & 2^{-n}\end{array}\right]$ where $n$ is an integer. Clearly $\Delta$ is a discrete subgroup and $\operatorname{det}(A d)$ is not constant on $\Delta$ so that $\left[w_{\frac{B}{\Delta}}\right] \neq 0$.

In Definition 41 and therefore in Proposition 21 we assumed completeness of $\varepsilon$. However this assumption can be dropped since we can choose $U$ sufficiently small in Definition 41 so that all 1-arrows with source and target in $U$ integrate to local diffeomorphisms defined on $U$. Therefore $[w]$ is in force also if $M$ is not 
complete. Many explicit and nontrivial examples of incomplete local Lie groups are constructed in [20] which fail to be globalizable. The example on pg. 49 in [20] is even simply connected, but such pathalogy can not occur if $M$ is complete in view of Corollary 19. We will postpone the study of these examples to some future work.

We will conclude this section with three remarks.

1) If $\varepsilon$ is complete, $\mathbf{A}$ forces $M$ to be a discrete quotient by Proposition 15 and hence analytic. Therefore $\mathbf{A}$ is a strong condition. On the other hand, we only need $\left.\operatorname{Tr} \mathcal{R}_{2}(\varepsilon)\right)=0$ to prove $d w=0$ and not $\mathbf{A}$. However, we are unable to attach a seperate geometric meaning to the condition $\operatorname{Tr} \mathcal{R}_{2}(\varepsilon)=0$.

2) We fix some $e \in M$ and a coordinate system around $e$ once and for all and write $\varepsilon_{j}^{i}(x, y)=\varepsilon_{a}^{i}(e, y) \varepsilon_{j}^{a}(x, e)$. Using the notation of [23], [24], we define a geometric object $w$ (not to be confused with the 1-form $w$ ) with components $w_{j}^{i}(x)$ over $\left(U, x^{i}\right)$ defined by $w_{j}^{i}(x) \stackrel{\text { def }}{=} \varepsilon_{j}^{i}(x, e)$ so that we have

$$
\varepsilon_{j}^{i}(x, y)=\bar{w}_{a}^{i}(y) w_{j}^{a}(x), \quad \bar{w}_{a}^{i}(y) w_{j}^{a}(y)=\delta_{j}^{i}
$$

Replacing derivatives with jet sections in the transformation rule $w_{j}^{i}(x)=$ $w_{a}^{i}(y) \frac{\partial y^{a}}{\partial x^{i}}$ gives

$$
w_{j}^{i}(x)=w_{a}^{i}(y) f_{i}^{a}(x)
$$

which describes the association of the object $w$ with the groupoid $\mathcal{U}_{1}$. Now (51) is taken as the basis of the Maurer-Cartan form in [23], [24] (see [23], pg. 212-221, 316-317, [24], pg. 27-32, 246-249). Substituting (51) into (40), a straightforward computation shows that the condition $\mathcal{R}(x, y)=0$ is equivalent to the second formula on pg. 28 in [24] which is

$$
\bar{w}_{j}^{a}(y) \bar{w}_{k}^{b}(y)\left(\frac{\partial w_{b}^{i}(y)}{\partial y^{a}}-\frac{\partial w_{a}^{i}(y)}{\partial y^{b}}\right)=\bar{w}_{j}^{a}(x) \bar{w}_{k}^{b}(x)\left(\frac{\partial w_{b}^{i}(x)}{\partial x^{a}}-\frac{\partial w_{a}^{i}(x)}{\partial x^{b}}\right)
$$

Thus both sides of (52) must be equal to the same constants $c_{j k}^{i}$. It is shown in [23], [24] that (51) is formally integrable if and only if (52) holds. Thus we see that (51) is a consequence of the existence of the splitting $\varepsilon$ and and the formal integrability of (51), a concept which plays a fundamental role in the works [23], [24], is equivalent to the condition $\mathcal{R}(\varepsilon)=0$.

Note that (16), (18) and (50) give

$$
\Gamma_{k j}^{i}(y) d y^{k}=\frac{\partial \bar{w}_{a}^{i}(y)}{\partial y^{k}} w_{j}^{a}(y) d y^{k}
$$

The 1-form on the LHS of (53) has only local meaning. On the other hand, $\bar{w}_{j}^{i}(y)=\varepsilon_{j}^{i}(y, e)$ uniquely determines some $g \in \mathcal{S}_{\varepsilon}$ in view of (41) and therefore we may write the RHS of $(53)$ as $(d g) g^{-1}$.

3) Let $M$ be parallelizable with the splitting $\varepsilon$. We fix $e \in\left(U, x^{i}\right)$, a tangent vecur $X_{e}$ at $e$, and define the $O D E$ for the unknown curve $x(t), 0 \leq t \leq \epsilon$, $x(0)=e$, by $\left(\varepsilon^{e, x}\right)_{*}\left(X_{e}\right)=\dot{x}(t)$ for $x \in U$. In coordinates, 


$$
\frac{d x^{i}}{d t}=\varepsilon_{a}^{i}(e, x) X^{a}, \quad 1 \leq i \leq n
$$

We call local solutions of (54) left 1-parameter curves. Differentiating (54) at $x=e$ and using (11), we get

$$
\frac{d^{2} x^{i}}{d t^{2}}(e)-\Gamma_{a b}^{i}(e) \frac{d x^{b}}{d t}(e) \frac{d x^{a}}{d t}(e)=0
$$

which are the equations for geodesics (note again the minus sign in (55) which arises from our convention of differentiating with respect to the second argument in (11)). Thus we can define left geodesic completeness of $M$. If $M$ is a local Lie group, then we can define locally also right 1-parameter curves and therefore 1parameter subgroups. It is an interesting problem to study the relations between these well known objects (which is done to some extent in [14], pg. 26-47, 97-131) and the relation between the concepts of completeness according to Definition 13 and geodesic completeness.

\section{Odd degree forms}

In this section we will define the higher order analogs of the closed 1-forms $\omega$ and $w$.

Turning back to $(29)$, let $X_{1}, \ldots, X_{k}$ be sections of $J_{1} T$. We define the $k$-form $\omega_{k}$ of $J_{1} T$ by

$$
\omega_{k}\left(X_{1}, \ldots, X_{k}\right) \stackrel{\text { def }}{=} \frac{1}{k} \sum_{\sigma \in S_{k}} \operatorname{sgn}(\sigma) \operatorname{Tr}\left(X_{\sigma(1)} \circ X_{\sigma(2)} \circ \ldots \circ X_{\sigma(k)}\right)
$$

Clearly, $\omega_{1}=\omega$.

Proposition $26 \omega_{2 m}=0, m \geq 1$

Proof: Consider the set $K$ of $k$-tuples $(\sigma(1), \sigma(2), \ldots, \sigma(k))$ where $\sigma$ is a permutation. We define an equivalence relation $\sim$ on $K:(\sigma(1), \sigma(2), \ldots, \sigma(k)) \sim$ $(\tau(1), \tau(2), \ldots, \tau(k))$ if $(\sigma(1), \sigma(2), \ldots, \sigma(k))=(\tau(i), \tau(i+1), \ldots \tau(k), \tau(1), \tau(2), \ldots$, $\tau(i-1))$ for some $i$, that is, two permutations are equivalent if they differ by a cyclic permutation. This equivalence relation is imposed by $\operatorname{Tr}(a b)=\operatorname{Tr}(b a)$. Now consider the formal sum

$$
\sum_{\sigma \in S_{k}} \operatorname{sgn}(\sigma)((\sigma(1), \sigma(2), \ldots, \sigma(k))
$$

The proof reduces to the following combinatorial statement: If $k$ is even and we identify the equivalent $k$-tuples in (51), then (51) vanishes. To prove this, we choose two odd integers $a, b$ with $k=a+b$. For some fixed $\sigma$, we have $\operatorname{sgn}(\sigma)(\sigma(1), \ldots, \sigma(a), \sigma(a+1), \ldots \sigma(a+b))=(-1)^{a b} \operatorname{sgn}(\sigma)(\sigma(a+1), \ldots \sigma(a+$ $b), \sigma(1), \ldots, \sigma(a))$ and all terms in $(51)$ cancel in pairs. 
Using 1 -arrows of $\varepsilon$ we can define $\varepsilon$-invariant $k$-forms on $M$. Following our convention for composition in Section 8, we will call these forms left invariant. Exterior derivative of a left invariant form need not be left invariant, but this is so if $\mathcal{R}(\varepsilon)=0$, that is, if $M$ is a local Lie group. Thus we get a complex whose cohomology $H^{*}\left(\mathfrak{X}_{\varepsilon}(M)\right)$ coincides with the Lie algebra cohomology of $\mathfrak{X}_{\varepsilon}(M)$. The left invariant forms can be localized at any point $p \in M$ and we obtain a complex at $p$ with cohomology $H_{p}^{*}\left(\mathfrak{X}_{\varepsilon}(M)\right)$. Since the evaluation map $e_{p}$ in (38) is an isomorphism of Lie algebras, it induces an isomorphism $e_{p}^{*}: H_{p}^{*}\left(\mathfrak{X}_{\varepsilon}(M)\right) \rightarrow H^{*}\left(\mathfrak{X}_{\varepsilon}(M)\right)$. If $q \in M$ is another point, there exists a unique $f \in \mathcal{S}_{\varepsilon}$ with $f(p)=q$. Since the local diffeomorphism $f$ commutes with the exterior derivative, it induces an isomorphism $f^{*}: H_{q}^{*}\left(\mathfrak{X}_{\varepsilon}(M)\right) \rightarrow H_{p}^{*}\left(\mathfrak{X}_{\varepsilon}(M)\right)$ and we obtain the commutative diagram

$$
\begin{aligned}
& H^{*}\left(\mathfrak{X}_{\varepsilon}(M)\right) \\
& e_{q}^{*} \nearrow \quad \nwarrow e_{p}^{*} \\
& H_{q}^{*}\left(\mathfrak{X}_{\varepsilon}(M)\right) \quad \stackrel{f^{*}}{\longrightarrow} \quad H_{p}^{*}\left(\mathfrak{X}_{\varepsilon}(M)\right)
\end{aligned}
$$

Now, we pull back the forms $\omega_{2 k+1}$ defined by (50) to $w_{2 k+1}$ on the local Lie group $M$. Let $X_{1}, X_{2}, \ldots . X_{k}$ be left invariant vector fields on $M$. Proposition 11, the derivation of (36) and (50) give the formula

$$
w_{k}\left(X_{1}, \ldots, X_{k}\right)=\frac{1}{k} \sum_{\sigma \in S_{k}} \operatorname{sgn}(\sigma) \operatorname{tr}\left(\operatorname{ad}\left(X_{\sigma(1)}\right) \circ \ldots \circ \operatorname{ad}\left(X_{\sigma(k)}\right)\right.
$$

As we noted at the end of Section $6, w_{1}$ is left invariant and (53) shows that $w_{2 k+1}$ is left invariant for all $k \geq 0$. Therefore the exterior derivative of $w_{2 k+1}$ can be computed algebraically.

Proposition $27 d w_{2 k+1}=0, k \geq 0$

Proof: If $h_{1} \circ h_{2} \circ \ldots \circ h_{k}$ is a composition of some linear maps and $\sigma \in S_{k}$, we define the composition

$$
\sigma *\left(h_{1} \circ h_{2} \circ \ldots \circ h_{k}\right) \stackrel{\text { def }}{=} h_{\sigma(1)} \circ h_{\sigma(2)} \circ \ldots \circ h_{\sigma(k)}
$$

and extend $*$ linearly over sums of compositions. Usind the notation (54), we now have (omitting the constant factors), $d w_{k}\left(X_{1}, \ldots, X_{k+1}\right)$ 


$$
\begin{aligned}
= & \sum_{i \leq j+1}(-1)^{i+j} w_{k}\left(\left[X_{i}, X_{j}\right], \ldots, \widehat{X}_{i}, \ldots, \widehat{X}_{j}, \ldots, X_{k+1}\right) \\
= & \sum_{i \leq j+1}(-1)^{i+j} \sum_{\sigma \in S_{k}} \operatorname{sgn}(\sigma) \operatorname{tr}\left[\sigma *\left(a d\left[X_{i}, X_{j}\right] \circ \ldots . . \circ a d X_{k+1}\right)\right] \\
= & \sum_{i \leq j+1}(-1)^{i+j} \sum_{\sigma \in S_{k}} \operatorname{sgn}(\sigma) \operatorname{tr}\left[\sigma *\left(\left[a d X_{i}, a d X_{j}\right] \circ \ldots . . \circ a d X_{k+1}\right)\right] \\
= & \sum_{i \leq j+1}(-1)^{i+j} \sum_{\sigma \in S_{k}} \operatorname{sgn}(\sigma) \operatorname{tr}\left[\sigma *\left(\left(a d X_{i} \circ a d X_{j}\right) \circ \ldots . . \circ a d X_{k+1}\right)\right] \\
& -\sum_{i \leq j+1}(-1)^{i+j} \sum_{\sigma \in S_{k}} \operatorname{sgn}(\sigma) \operatorname{tr}\left[\sigma *\left(\left(a d X_{j} \circ a d X_{i}\right) \circ \ldots . . \circ a d X_{k+1}\right)\right]
\end{aligned}
$$

Now each term in the last sum in (55) contains a composition of $k$ linear maps. The value of trace does not change if we apply some $k$-cycle to its arguments. If $k$ is odd and $\sigma \in S_{k}$ is a $k$-cycle, then $\operatorname{sgn}(\sigma)=1$. Using these facts, a straightforward computation shows that all terms in the last sum in (55) cancel in pairs. We will omit the details.

Observe that (53) and Proposition 27 define odd order characteristic classes in the Lie algebra cohomology of any abstract Lie algebra $\mathfrak{g}$. We will denote these classes by $\left[w_{2 k+1}\right]_{\mathfrak{g}} \in H^{2 k+1}(\mathfrak{g})$. In fact, one can replace $a d$ with any representation $\rho: \mathfrak{g} \rightarrow V$ and still define these classes which however will depend on the representation as in [6]. We will not pursue this more general approach here.

If $\Delta \subset G$ a discrete subgroup, then $\left[w_{2 k+1}\right]_{d R} \in H^{2 k+1}(M, \mathbb{R})$ is defined in view of Proposition 27. We also have $\left[w_{2 k+1}\right]_{\mathfrak{X}(\varepsilon)} \in H^{2 k+1}\left(\mathfrak{X}_{\varepsilon}(M)\right)$. It is worthwhile to observe that these cohomology classes are intrinsic characteristic classes of the local Lie group $M$ and are defined regardless of any foliation. If $\left[w_{2 k+1}\right]_{\mathfrak{X}(\varepsilon)}=0$, then clearly $\left[w_{2 k+1}\right]_{d R}=0$ but the converse is false as shown by our noncompact example in Section 8: $\left[w_{1}\right]_{\mathfrak{X}(\varepsilon)}=0$ if and only if $w_{1}=0$ whereas we may have $\left[w_{1}\right]_{d R}=0$ with nonconstant primitive $-\log \left(\operatorname{det} A d_{e}\right)$ and therefore $w_{1} \neq 0$.

Now let $G=S L(2, \mathbb{R}), \Delta \subset G$ a cocompact discrete subgroup and $\mathcal{F}$ the codimension one foliation on $M$ defined by the left invariant vector fields $X=$ $\left[\begin{array}{ll}0 & 1 \\ 0 & 0\end{array}\right], H=\left[\begin{array}{cc}1 & 0 \\ 0 & -1\end{array}\right]$. Let $G V(\mathcal{F}) \in H^{3}(M, \mathbb{R})$ denote the Godbillon-Vey class of this foliation (see [4], pg. 62-64 for details). To distinguish between $w_{2 k+1}$ on the local Lie groups $G$ and $M=G / \Delta$, we will use the notations $w_{2 k+1}^{G}$ and $w_{2 k+1}^{M}$.

Proposition $28\left[w_{3}^{M}\right]_{d R}=G V(\mathcal{F})$

Proof: It suffices to check that $w_{3}^{G}$ is a nonzero multiple of an invariant volume form on $S L(2, \mathbb{R})$. Let $X, H, Y$ be the basis of left invariant vector fields on $S L(2, \mathbb{R})$ where $Y=\left[\begin{array}{ll}0 & 0 \\ 1 & 0\end{array}\right]$ and $X, H$ are as above. We define the 
invariant volume form $\nu$ on $S L(2, \mathbb{R})$ by $\nu=X^{*} \Lambda H^{*} \Lambda Y^{*}$ where $X^{*}, H^{*}, Y^{*}$ are the dual 1-forms. On the other hand, (53) gives

$$
\begin{aligned}
w_{3}^{G}(X, H, Y)= & \frac{1}{3}\{\operatorname{Tr}(a d X \circ a d H \circ a d Y)+\operatorname{Tr}(a d Y \circ a d X \circ a d H) \\
& +\operatorname{Tr}(a d H \circ a d Y \circ a d X)-\operatorname{Tr}(a d X \circ A d Y \circ a d H) \\
& -\operatorname{Tr}(a d H \circ a d X \circ a d Y)-\operatorname{Tr}(a d Y \circ a d H \circ a d X)\} \\
= & \operatorname{Tr}\{a d X \circ a d H \circ a d Y)-(a d X \circ A d Y \circ a d H)\}
\end{aligned}
$$

We have

$$
[H, X]=2 X, \quad[H, Y]=-2 Y, \quad[X, Y]=H
$$

Using (53) and (55), an easy computation gives $w_{3}^{G}(X, H, Y)=-8=-8 v(X, H, Y)$ and therefore $w_{3}^{G}=-8 \nu$.

Proposition 28 gives rise to a natural and, we believe, intriguing question. Let $H \subset G$ be a subgroup of codimension $k$. The cosets of $H$ foliate $G$ and this foliation descends to a foliation $\mathcal{F}$ on $G / \Delta$ with Godbillon-Vey class $G V(\mathcal{F}) \in$ $H^{2 k+1}(G / \Delta, \mathbb{R})$. On the other hand we also have $\left[w_{2 k+1}\right]_{d R} \in H^{2 k+1}(G / \Delta, \mathbb{R})$ which is defined even if $G$ does not have any subgroup of codimension $k$.

Q : What is the relation between $G V(\mathcal{F})$ and $\left[w_{2 k+1}\right]_{d R}$ ?

Since $\left[w_{1}\right]_{d R}$ is an obstruction to globalizability, it is natural to expect that this is true for all $\left[w_{2 k+1}\right]_{d R}$, that is, $\left[w_{2 k+1}\right]_{d R}=0$ if $M$ is globalizable as we first conjectured. However, the following example communicated to us by the referee shows that this conjecture is false: let $G=S O(3, \mathbf{R})$ with the Lie algebra $\mathfrak{s u}(3, \mathbb{R})$ generated by

$$
A=\left[\begin{array}{ccc}
0 & 1 & 0 \\
-1 & 0 & 0 \\
0 & 0 & 0
\end{array}\right], B=\left[\begin{array}{ccc}
0 & 0 & 1 \\
0 & 0 & 0 \\
-1 & 0 & 0
\end{array}\right], C=\left[\begin{array}{ccc}
0 & 0 & 0 \\
0 & 0 & 1 \\
0 & -1 & 0
\end{array}\right]
$$

We have

$$
[A, C]=B,[B, A]=C,[C, B]=A
$$

As in the proof of Proposition 28, (57) together with $(54)$ gives $w_{3}^{G}(A, B, C)=$ $16 \neq 0$. Therefore $w_{3}^{G}$ is again a volume form and is not exact since $G$ is compact.

The above examples $\mathfrak{s l}(2, \mathbb{R}), \mathfrak{s u}(3, \mathbb{R})$ are particular instances of a general phenomenon. To see this, we continue to compute with the last equality in (62) by replacing $X, H, Y$ with arbitrary elements $x, y, z$ in some abstract Lie algebra $\mathfrak{g}:$

$$
\begin{aligned}
w_{3}(x, y, z) & =\operatorname{Tr}\{a d x \circ a d y \circ a d z)-(a d x \circ a d z \circ a d y)\} \\
& =\operatorname{Tr}\{a d x \circ(a d y \circ a d z-a d z \circ a d y) \\
& =\operatorname{Tr}\{a d x \circ a d[y, z]\} \\
& =\kappa(x,[y, z])
\end{aligned}
$$


where $\kappa$ is Killing form.

Proposition 29 i) $\left[w_{3}\right]_{\mathfrak{g}} \neq 0$ if $\mathfrak{g}$ is semisimple.

ii) $w_{3}=0$ if and only if $\mathfrak{g}$ is solvable.

Proof: i) This follows from (66) and the proof of Theorem 21.1 in [5].

ii) This is again (66) together with the well known Cartan's criterion for solvability.

The proof of Theorem 21.1 in [5] shows that $w_{3}$ is not only left invariant but also right invariant, that is, invariant, and this is easy to show for all $w_{2 k+1}$ so that Proposition 27 is a consequence.

In view of Proposition 29, $\left[w_{3}\right]_{\mathfrak{g}}$ may be interpreted as an obstruction to the solvability of the Lie algebra $\mathfrak{g}$. Recalling that the concept of solvability originated in Galois theory in an attempt to solve polynomial equations by radicals, this interpretation is clearly far from being satisfactory unless we clarify what is to be solved in the present framework.

We would like to conclude with an amusing speculation about an intriguing relation between local Lie groups and Poincare conjecture and a related question. Let $M$ be a compact, simply connected 3-manifold together with its unique differentiable structure. It is well known that $M$ is parallelizable and therefore admits splittings by Lemma 3. If $M$ admits a flat splitting, then it will be a local Lie group and therefore a Lie group by Lemma 14 and Corollary 19, but $S^{3}$ is the only compact, simply connected 3 -dimensional Lie group. Therefore, Poincare Conjecture (now a theorem thanks to the deep works of Hamilton and Perelman) is equivalent to the assertion that $M$ admits a flat splitting. This fact suggests, we believe, that Poincare Conjecture may have a short and metric-independent proof.

The characteristic classes $\left[w_{2 k+1}\right]_{d R}$ are secondary since they arise when $M$ admits a splitting $\varepsilon$ with $\mathcal{R}(\varepsilon)=0$. The natural question arises whether there exist any primary classes in the present framework.

Q : Are there any obstructions to the existence of a flat splitting on a parallelizable manifold?

Acknowledgements: We are indebted to the anonymous referee for giving the counterexample $S O(3, \mathbf{R})$ to our conjecture that $\left[w_{2 k+1}\right]_{d R}$ is an obstruction to globalizability also for $k \geq 1$, and for pointing out that our "primary classes" vanish. We are also indebted to Gregor Weingart for finding a serious mistake in the original version of this paper and for his stimulating remarks. Last but not least, we express our heartfelt gratitude to P. J. Olver for his kind support and encouragement for this work.

\section{References}

[1] E. Abadoğlu, E. Ortaçgil, F. Öztürk: Klein geometries, parabolic geometries and differential equations of finite type, J. Lie Theory 18 (2008) no.1, $67-82$ 
[2] A.D. Blaom: Geometric structures as deformed infinitesimal symmetries, Trans. Amer. Math. Soc. 358 (2006), no.8, 3651-3671(electronic)

[3] A.D. Blaom: Lie algebroids and Cartan's method of equivalence, arXiv: math/0509071v2 [math.DG]

[4] R. Bott: Lectures on characteristic classes and foliations. Notes by Lawrence Conlon, with two appendices by J.Stasheff. Lectures on algebraic and differential topology (Second Latin American School in Math., Mexico City, 1971, pp.1-94. Lecture Notes in Math., Vol. 279, Springer, Berlin, 1972

[5] C. Chevalley, S. Eilenberg: Cohomology theory of Lie groups and Lie algebras, Trans. Amer. Math. Soc. 63 (1948), 85-124

[6] M. Crainic: Differentiable and algebroid cohomology, Van Est isomorphisms, and characteristic classes, Comment. Math. Helv. 78 (2003), no.4, 681-721

[7] M. Crainic, R.L. Fernandes: Secondary characteristic classes of Lie algebroids, Quantum field theory and noncommutative geometry, 157-176, Lecture Notes in Phys., 662, Springer, Berlin, 2005

[8] M. Crainic, I. Moerdijk: Deformations of Lie brackets: cohomological aspects, arXiv:math / 0403434

[9] P.A. Damianou, R.L. Fernandes: Integrable hierarchies and the modular class, Ann. Inst. Fourier (Grenoble) 58 (2008), no. 1, 107-137

[10] S. Evens, J.H. Lu, A. Weinstein: Transverse measures, the modular class and a cohomology pairing for Lie algebroids, Quart. J. Math., Oxford Ser. (2), 50 (1999), no. 200, 417-436

[11] R.L. Fernandes: Lie algebroids, holonomy and characteristic classes, Adv. Math. 170 (2002), no. 1, 119-179

[12] R.B. Gardner: The Method of Equivalence and its Applications, SIAM, Philedelphia, 1989

[13] P. Griffiths: On Cartan's method of Lie groups and moving frames as applied to uniqueness and existence questions in differential geometry, Duke Math. J.. 41 (1974), 775-814

[14] S. Helgason: Differential geometry, Lie groups, and symmetric spaces. Corrected reprint of the 1978 original, Graduate Studies in Mathematics, 34. AMS, Providence, RI, 2001

[15] F.W. Kamber, P. Tondeur: Foliated bundles and characteristic classes. Lecture Notes in Mathematics, Vol. 493. Springer-Verlag, Berlin-New York, 1975

[16] S. Kobayashi, K. Nomizu: Foundations of Differential Geometry. Vol. I, Interscience Publishers, a division of John Wiley \& Sons, New York-London, 1963

[17] A. Kumpera, D.C. Spencer: Lie Equations. Vol. I: General Theory, Annals of Mathematics Studies, No. 73, Princeton University Press, Princeton, N.J, Univertsity of Tokyo Press, Tokyo, 1972

[18] K. MacKenzie: Lie Groupoids and Lie Algebroids in Differential Geometry, London Mathematical Society Lecture Note Series, 124, Cambridge University Press, Cambridge, 1987 
[19] K. MacKenzie: General Theory of Lie Groupoids and Lie Algebroids, London Mathematical Society Lecture Note Series, 213, Cambridge University Press, Cambridge, 2005

[20] P.J. Olver: Non-associative local Lie groups, J. Lie 6 (1996), no.1, 23-51

[21] E. Ortacgil: The heritage of S.Lie and F.Klein: Geometry via transformation groups, arXiv:math / 0604223, April 2006

[22] E. Ortaçgil: Pre-homogeneous geometric structures and their curvatures, in progress

[23] J.F. Pommaret: Systems of Partial Differential Equations and Lie Pseudo groups. With a preface by Andre Lichnerowicz, Mathemetics and its Applications, 14. Gordon\&Breach Science Publishers, New York, 1978

[24] J.F. Pommaret: Partial Differential Equations and Group Theory. New Perspectives for Applications, Mathematics and its Applications, 293. Kluwer Academic Publishers Group, Dordrecht, 1994

[25] L.S. Pontryagin: Selected works. Vol.2, Topological groups, Edited and with a preface by R.V. Gamkrelidze, translated by Arlen Brown007 (90a:01106) Pontryagin, L. S. Selected works. Vol. 2. Topological groups, with a preface by R. V. Gamkrelidze, Classics of Soviet Mathematics, Gordon \& Breach Science Publishers, New York, 1986

[26] R.W. Sharpe: Differential Geometry, Cartan's Generalization of Klein's Erlangen Program, with a foreword by S.S.Chern, Graduate Texts in Mathematics, 166. Springer-Verlag, New York, 1997

[27] O. Veblen: Invariants of quadratic differential forms, Cambridge University Press, 1962

[28] G. Weingart: Holonomic and semi-holonomic geometries, Global Analysis and Harmonic Analysis (Marseille-Luminy, 1999), 307-328, Semin. Congr., 4, Soc. Math. France, Paris, 2000

[29] A. Weinstein: The modular automorphism group of a Poisson manifold, J. Geom. Phys., 23 (1997), no. 3-4, 379-394

Ender Abadoğlu, Yeditepe University, Mathematics Department, 81120, Kayışdağı, İstanbul, Türkiye

e-mail: eabadoglu@yeditepe.edu.tr

Ercüment Ortaçgil, Boğaziçi University, Mathematics Department, Bebek, 34342, İstanbul, Türkiye

e-mail: ortacgil@boun.edu.tr 